\title{
GENERIC EXISTENCE OF SOLUTIONS OF NONCONVEX OPTIMAL CONTROL PROBLEMS
}

\author{
ALEXANDER J. ZASLAVSKI
}

Received 8 December 2003

The Tonelli existence theorem in the calculus of variations and its subsequent modifications were established for integrands $f$ which satisfy convexity and growth conditions. In 1996, the author obtained a generic existence and uniqueness result (with respect to variations of the integrand of the integral functional) without the convexity condition for a class of optimal control problems satisfying the Cesari growth condition. In this paper, we survey this result and its recent extensions, and establish several new results in this direction.

\section{Introduction}

The Tonelli existence theorem in the calculus of variations $[17,18]$ and its subsequent generalizations and extensions (e.g., $[5,11,14,16])$ are based on two fundamental hypotheses concerning the behavior of the integrand as a function of the last argument (derivative): one is that the integrand should grow superlinearly at infinity and the other is that it should be convex (or exhibit a more special convexity property in case of a multiple integral with vector-valued functions) with respect to the last variable. Moreover, certain convexity assumptions are also necessary for properties of lower semicontinuity of integral functionals which are crucial in most of the existence proofs, although there are some interesting theorems without convexity (see [5, Chapter 16] and $[2,4,13]$ ).

In 1996, the author showed that the convexity condition is not needed generically, and not only for the existence but also for the uniqueness of a solution and even for well-posedness of the problem (with respect to some natural topology in the space of integrands). This result was published in [22]. Instead of considering the existence of a solution for a single integrand $f$, we investigated it for a space of integrands and showed that a unique solution exists for most of the integrands in the space. This approach has already been successfully applied in the theory of dynamical systems (see $[6,7,15])$, as well as in the calculus of variations (see, e.g., $[1,19,21]$ ). Interesting generic existence results were obtained for particular cases of variational problems $[3,12]$. In [3, 12] were studied integrands of the form $L(x, v)=g(x)+h(v)$ where $h$ is nonconvex and $x$ is scalarvalued. It was shown in [3] that the set $\mathscr{D}$ of all continuous functions $g$ such that for any 
$h$ the corresponding variational problem has a solution is an everywhere dense subset of $C\left(\mathbb{R}^{1}\right)$ equipped with the topology of uniform convergence on bounded subsets. In [12] it was established that the set $\mathscr{D}$ is of the first category in $C\left(\mathbb{R}^{1}\right)$. In [22] the same approach allowed us to establish the generic existence of solutions for a large class of optimal control problems without convexity assumptions.

More precisely, in [22] we considered a class of optimal control problems (with the same system of differential equations, the same functional constraints, and the same boundary conditions) which is identified with the corresponding complete metric space of cost functions (integrands), say $\mathscr{F}$. We did not impose any convexity assumptions. These integrands are only assumed to satisfy the Cesari growth condition. The main result in [22] establishes the existence of an everywhere dense $G_{\delta}$-set $\mathscr{F}^{\prime} \subset \mathscr{F}$ such that for each integrand in $\mathscr{F}^{\prime}$, the corresponding optimal control problem has a unique solution.

The next step in this area of research was done in [10]. There we introduced a general variational principle having its prototype in the variational principle of Deville et al. [8]. A generic existence result in the calculus of variations without convexity assumptions was then obtained as a realization of this variational principle. It was also shown in [10] that some other generic well-posedness results in optimization theory known in the literature and their modifications are obtained as a realization of this variational principle. Note that the generic existence result in [10] was established for variational problems but not for optimal control problems and that the topologies in the spaces of integrands in [10, 22] are different.

In [20] we suggested a modification of the variational principle in [10] and applied it to classes of optimal control problems with various topologies in the corresponding spaces of integrands. As a realization of this principle, we established, generic existence results for classes of optimal control problems in which constraint maps are also subject to variations as well as the cost functions. More precisely, we established generic existence results for classes of optimal control problems (with the same system of differential equations, the same boundary conditions, and without convexity assumptions) which are identified with the corresponding complete metric spaces of pairs $(f, U)$ (where $f$ is an integrand satisfying the Cesari growth condition and $U$ is a constraint map) endowed with some natural topology. We showed that for a generic pair $(f, U)$ the corresponding optimal control problem has a unique solution.

In this paper, we discuss the results of $[20,22]$ and establish extensions of the main result of [20].

\section{Bolza problems of optimal control}

Let $-\infty<T_{1}<T_{2}<\infty$, let $A \subset\left[T_{1}, T_{2}\right] \times \mathbb{R}^{n}$ be a closed subset of the $t x$-space $\mathbb{R}^{n+1}$, and let $A(t)$ denote its sections, that is,

$$
A(t)=\left\{x \in \mathbb{R}^{n}:(t, x) \in A\right\}, \quad t \in\left[T_{1}, T_{2}\right] .
$$

For every $(t, x) \in A$, let $U(t, x)$ be a given subset of the $u$-space $\mathbb{R}^{m}, x=\left(x_{1}, \ldots, x_{n}\right), u=$ $\left(u_{1}, \ldots, u_{m}\right)$. 
Let $M$ denote the set of all $(t, x, u)$ with $(t, x) \in A, u \in U(t, x)$, and let $B_{1}, B_{2} \subset \mathbb{R}^{n}$ be closed. We assume that the set $M$ is closed and $A(t) \neq \varnothing$ for every $t \in\left[T_{1}, T_{2}\right]$. Let $H(t, x, u)=\left(H_{1}, \ldots, H_{n}\right)$ be a given continuous function defined on $M$.

We say that a pair $x:\left[T_{1}, T_{2}\right] \rightarrow \mathbb{R}^{n}, u:\left[T_{1}, T_{2}\right] \rightarrow \mathbb{R}^{m}$ is admissible if $x=\left(x_{1}, \ldots, x_{n}\right)$ is an absolutely continuous (a.c.) function, $u=\left(u_{1}, \ldots, u_{m}\right)$ is a measurable function, and the following relations hold:

$$
\begin{gathered}
x(t) \in A(t), \quad t \in\left[T_{1}, T_{2}\right], \quad x\left(T_{i}\right) \in B_{i}, \quad i=1,2, \\
u(t) \in U(t, x(t)), \quad x^{\prime}(t)=H(t, x(t), u(t)), \quad t \in\left[T_{1}, T_{2}\right] \text { a.e. }
\end{gathered}
$$

Denote by $\Omega$ the set of all admissible pairs $(x, u)$. We suppose that $\Omega \neq \varnothing$.

In this section, we are concerned with the existence of the minimum in $\Omega$ of the functional

$$
\int_{T_{1}}^{T_{2}} f(t, x(t), u(t)) d t+h\left(x\left(T_{1}\right), x\left(T_{2}\right)\right),
$$

where $h: B_{1} \times B_{2} \rightarrow \mathbb{R}^{1}$ is a lower semicontinuous bounded below function, and $f$ belongs to a space of functions described below.

Denote by $C_{l}\left(B_{1} \times B_{2}\right)$ the set of all lower semicontinuous bounded below functions $h: B_{1} \times B_{2} \rightarrow \mathbb{R}^{1}$, and denote by $C\left(B_{1} \times B_{2}\right)$ the set of all continuous functions $h \in C_{l}\left(B_{1} \times\right.$ $\left.B_{2}\right)$. For the set $C_{l}\left(B_{1} \times B_{2}\right)$, we consider, the uniformity which is determined by the base

$$
E_{0}(\epsilon)=\left\{\left(h_{1}, h_{2}\right) \in C_{l}\left(B_{1} \times B_{2}\right) \times C_{l}\left(B_{1} \times B_{2}\right):\left|h_{1}(z)-h_{2}(z)\right| \leq \epsilon, z \in B_{1} \times B_{2}\right\},
$$

where $\epsilon>0$. It is easy to verify that the uniform space $C_{l}\left(B_{1} \times B_{2}\right)$ is metrizable and complete, and $C\left(B_{1} \times B_{2}\right)$ is a closed subset of $C_{l}\left(B_{1} \times B_{2}\right)$. We consider the topological space $C\left(B_{1} \times B_{2}\right) \subset C_{l}\left(B_{1} \times B_{2}\right)$ which has the relative topology.

Denote by $\mathfrak{M}_{l}$ the set of all lower semicontinuous functions $f: M \rightarrow \mathbb{R}^{1}$ which satisfy the following growth condition.

For each $\epsilon>0$, there exists an integrable scalar function $\psi_{\epsilon}(t) \geq 0, t \in\left[T_{1}, T_{2}\right]$, such that $|H(t, x, u)| \leq \psi_{\epsilon}(t)+\epsilon f(t, x, u)$ for each $(t, x, u) \in M$.

This growth condition proposed by Cesari (see [5]) and its equivalents and modifications are rather common in the literature.

Denote by $\mathfrak{M}_{c}$ the set of all continuous functions $f \in \mathfrak{M}_{l}$. For $N, \epsilon>0$, we set

$$
\begin{aligned}
E(N, \epsilon)= & \left\{(f, g) \in \mathfrak{M}_{l} \times \mathfrak{M}_{l}:|f(t, x, u)-g(t, x, u)| \leq \epsilon((t, x, u) \in M,|x|,|u| \leq N),\right. \\
& |f(t, x, u)-g(t, x, u)| \leq \epsilon+\epsilon \sup \{|f(t, x, u)|,|g(t, x, u)|\}((t, x, u) \in M)\}
\end{aligned}
$$

We can show in a straightforward manner that for the set $\mathfrak{M}_{l}$ there exists the uniformity which is determined by the base $E(N, \epsilon), N, \epsilon>0$. It is easy to verify that the uniform space $\mathfrak{M}_{l}$ is metrizable and complete. Clearly $\mathfrak{M}_{c}$ is a closed subset of $\mathfrak{M}_{l}$. We consider 
the topological space $\mathfrak{M}_{c} \subset \mathfrak{M}_{l}$ which has the relative topology, and the spaces

$$
\mathfrak{A}_{l}=\mathfrak{M}_{l} \times C_{l}\left(B_{1} \times B_{2}\right), \quad \mathfrak{A}_{c}=\mathfrak{M}_{c} \times C\left(B_{1} \times B_{2}\right)
$$

which have the product topology.

We consider the functionals of the form

$$
I^{(f, h)}(x, u)=\int_{T_{1}}^{T_{2}} f(t, x(t), u(t)) d t+h\left(x\left(T_{1}\right), x\left(T_{2}\right)\right),
$$

where $(x, u) \in \Omega, f \in \mathfrak{M}_{l}$ and $h \in C_{l}\left(B_{1} \times B_{2}\right)$.

For each $f \in \mathfrak{M}_{l}$, and each $h \in C_{l}\left(B_{1} \times B_{2}\right)$, we consider the problem of the absolute minimum

$$
I^{(f, h)}(x, u) \longrightarrow \min , \quad(x, u) \in \Omega,
$$

and set

$$
\mu(f, h)=\inf \left\{I^{(f, h)}(x, u):(x, u) \in \Omega\right\}
$$

It is easy to see that

$$
\mu(f, h)>-\infty \quad \text { for each } f \in \mathfrak{M}_{l} \text {, each } h \in C_{l}\left(B_{1} \times B_{2}\right)
$$

Denote by mes $(E)$ the Lebesgue measure of a measurable set $E \subset \mathbb{R}^{k}$ and denote by $|\cdot|$ the Euclidean norm in $\mathbb{R}^{k}$. Define

$$
\mathfrak{A}_{l, \mathrm{reg}}=\left\{(f, h) \in \mathfrak{A}_{l}: \mu(f, h)<\infty\right\}, \quad \mathfrak{A}_{c, \mathrm{reg}}=\mathfrak{A}_{l, \mathrm{reg}} \cap \mathfrak{A}_{c} .
$$

Denote by $\overline{\mathfrak{A}}_{l \text {,reg }}$ the closure of $\mathfrak{A}_{l \text {,reg }}$ in $\mathfrak{A}_{l}$, and by $\overline{\mathfrak{A}}_{c \text {,reg }}$ the closure of $\mathfrak{A}_{c \text {,reg }}$ in $\mathfrak{A}_{c}$. For each $h \in C_{l}\left(B_{1} \times B_{2}\right)$, we define

$$
\mathfrak{M}_{l, \mathrm{reg}}^{h}=\left\{f \in \mathfrak{M}_{l}: \mu(f, h)<\infty\right\}, \quad \mathfrak{M}_{c, \text { reg }}^{h}=\left\{f \in \mathfrak{M}_{c}: \mu(f, h)<\infty\right\}
$$

Denote by $\overline{\mathfrak{M}}_{l \text {,reg }}^{h}$ the closure of $\mathfrak{M}_{l, \text { reg }}^{h}$ in $\mathfrak{M}_{l}$, and by $\overline{\mathfrak{M}}_{c, \text { reg }}^{h}$ the closure of $\mathfrak{M}_{c, \text { reg }}^{h}$ in $\mathfrak{M}_{c}$.

We showed in [22] that $\mathfrak{A}_{l \text {,reg }}$ is an open subset of $\mathfrak{A}_{l}, \mathfrak{A}_{c \text {,reg }}$ is an open subset of $\mathfrak{A}_{c}$, and for each $h \in C_{l}\left(B_{1} \times B_{2}\right), \mathfrak{M}_{l, \text { reg }}^{h}$ is an open subset of $\mathfrak{M}_{l}$, and $\mathfrak{M}_{c \text {,reg }}^{h}$ is an open subset of $\mathfrak{M}_{c}$. We consider the topological subspaces $\overline{\mathfrak{A}}_{c \text {,reg }} \subset \mathfrak{A}_{c}, \overline{\mathfrak{A}}_{l \text {,reg }} \subset \mathfrak{A}_{l}, \overline{\mathfrak{M}}_{l, \text { reg }}^{h} \subset \mathfrak{M}_{l}, \overline{\mathfrak{M}}_{c, \text { reg }}^{h} \subset$ $\mathfrak{M}_{c}\left(h \in C_{l}\left(B_{1} \times B_{2}\right)\right)$ with the relative topology.

In [22] we established the following results which show that generically the optimal control problem considered in this section has a unique solution.

THeOREM 2.1. There exist a set $\mathfrak{F}_{l} \subset \overline{\mathfrak{A}}_{l \text {,reg }}$ which is a countable intersection of open everywhere dense subsets of $\overline{\mathfrak{A}}_{l, \text { reg }}$, and a set $\mathfrak{F}_{c} \subset \overline{\mathfrak{A}}_{c, \text { reg }} \cap \mathfrak{F}_{l}$ which is a countable intersection of 
open everywhere dense subsets of $\overline{\mathfrak{A}}_{c, \text { reg }}$, such that for each $(f, h) \in \mathfrak{F}_{l}$ the following assertions hold:

(1) $\mu(f, h)<\infty$ and there exists a unique $\left(x^{(f, h)}, u^{(f, h)}\right) \in \Omega$ for which

$$
I^{(f, h)}\left(x^{(f, h)}, u^{(f, h)}\right)=\mu(f, h) .
$$

(2) for each $\epsilon>0$, there exist a neighborhood $U$ of $(f, h)$ in $\mathfrak{A}_{l}$ and a number $\delta>0$ such that for each $(g, \xi) \in U$ and each $(x, u) \in \Omega$ satisfying $I^{(g, \xi)}(x, u) \leq \mu(g, \xi)+\delta$, the following relation holds:

$$
\operatorname{mes}\left\{t \in\left[T_{1}, T_{2}\right]:\left|x(t)-x^{(f, h)}(t)\right|+\left|u(t)-u^{(f, h)}(t)\right| \geq \epsilon\right\} \leq \epsilon .
$$

Note that by the Baire category theorem, the set $\mathfrak{F}_{l}$ is nonempty and in fact everywhere dense in $\overline{\mathfrak{A}}_{l, \text { reg }}$.

Theorem 2.2. Let $\eta \in C_{l}\left(B_{1} \times B_{2}\right)$ be fixed and let $\mathfrak{F}_{l}, \mathfrak{F}_{c}$ be as guaranteed in Theorem 2.1 . Then there exist a set $\mathfrak{F}_{l}^{\eta} \subset \overline{\mathfrak{M}}_{l, \text { reg }}^{\eta}$ which is a countable intersection of open everywhere dense subsets of $\overline{\mathfrak{M}}_{l, \text { reg }}^{\eta}$, and a set $\mathfrak{F}_{c}^{\eta} \subset \overline{\mathfrak{M}}_{c, \text { reg }}^{\eta} \cap \mathfrak{F}_{l}^{\eta}$ which is a countable intersection of open everywhere dense subsets of $\overline{\mathfrak{M}}_{c, \text { reg, }}^{\eta}$ such that

$$
\mathfrak{F}_{l}^{\eta} \times\{\eta\} \subset \mathfrak{F}_{l}
$$

\section{Optimal control problems with multiple integrals}

Let $\mathfrak{K}$ be a bounded domain in $\mathbb{R}^{m}$ where $m>1$, let

$$
W^{1,1}(\mathfrak{K})=\left\{u \in L^{1}(\mathfrak{K}): \frac{\partial u}{\partial x_{j}} \in L^{1}(\mathfrak{K}), j=1, \ldots, m\right\},
$$

and let $W_{0}^{1,1}(\mathfrak{K})$ be the closure of $C_{0}^{\infty}(\mathfrak{K})$ in $W^{1,1}(\mathfrak{K})$, where $C_{0}^{\infty}(\mathfrak{K})$ is the space of smooth functions $u: \mathfrak{K} \rightarrow \mathbb{R}^{1}$ with compact support in $\mathfrak{K}$.

For a function $u=\left(u_{1}, \ldots, u_{n}\right)$, where $u_{i} \in W^{1,1}(\mathfrak{K}), i=1, \ldots, n$, we set

$$
\nabla u_{i}=\left(\frac{\partial u_{i}}{\partial x_{j}}\right)_{j=1}^{m}, \quad i=1, \ldots, n, \nabla u=\left(\nabla u_{i}\right)_{i=1}^{n} .
$$

Assume that $A \subset \mathfrak{K} \times \mathbb{R}^{n}$, for each $\omega \in \mathfrak{K}$,

$$
A(\omega)=\left\{x \in \mathbb{R}^{n}:(\omega, x) \in A\right\} \neq \varnothing,
$$

and for every $(\omega, x) \in A, U(\omega, x)$ is a given subset of $u$-space $\mathbb{R}^{N}$.

Let $M$ denote the set of all $(\omega, x, u)$ with $(\omega, x) \in A, u \in U(\omega, x)$. We assume that the set $M$ is a closed subset of the space $\mathfrak{K} \times \mathbb{R}^{n} \times \mathbb{R}^{N}$ with the product topology. Let $H(\omega, x, u)$ be a given continuous function defined on $M$ such that

$$
H(\omega, x, u)=\left(H_{i}\right)_{i=1}^{n}, \quad H_{i}=\left(H_{i, j}\right)_{j=1}^{m}, \quad i=1, \ldots, n,
$$

and let $\theta^{*}=\left(\theta_{i}^{*}\right)_{i=1}^{n} \in\left(W^{1,1}(\mathfrak{K})\right)^{n}$ be fixed. 
We say that a pair $x=\left(x_{1}, \ldots, x_{n}\right) \in\left(W^{1,1}(\mathfrak{K})\right)^{n}, u=\left(u_{1}, \ldots, u_{N}\right): \mathfrak{K} \rightarrow \mathbb{R}^{N}$ is admissible if $u$ is measurable and the following relations hold:

$$
\begin{gathered}
x(\omega) \in A(\omega), \quad \omega \in \mathfrak{K} \text { a.e., } \quad u(\omega) \in U(\omega, x(\omega)), \quad \omega \in \mathfrak{K} \text { a.e., } \\
\nabla x(\omega)=H(\omega, x(\omega), u(\omega)), \quad \omega \in \mathfrak{K} \text { a.e., } \quad x-\theta^{*} \in\left(W_{0}^{1,1}(\mathfrak{K})\right)^{n} .
\end{gathered}
$$

Denote by $\Omega$ the set of all admissible pairs $(x, u)$. We suppose that $\Omega \neq \varnothing$.

Denote by $\mathfrak{M}_{l}$ the set of all lower semicontinuous functions $f: M \rightarrow \mathbb{R}^{1}$ which satisfy the following growth condition.

For each $\epsilon>0$ there exists an integrable scalar function $\psi_{\epsilon}(\omega) \geq 0, \omega \in \mathfrak{K}$, such that $|H(\omega, x, u)| \leq \psi_{\epsilon}(\omega)+\epsilon f(\omega, x, u)$ for all $(\omega, x, u) \in M$.

Denote by $\mathfrak{M}_{c}$ the set of all continuous functions $f \in \mathfrak{M}_{l}$. For $N, \epsilon>0$, we set

$$
\begin{aligned}
E(N, \epsilon)=\{ & (f, g) \in \mathfrak{M}_{l} \times \mathfrak{M}_{l}:|f(\omega, x, u)-g(\omega, x, u)| \leq \epsilon((\omega, x, u) \in M,|x|,|u| \leq N), \\
& |f(\omega, x, u)-g(\omega, x, u)| \leq \epsilon+\epsilon \sup \{|f(\omega, x, u)|,|g(\omega, x, u)|\}(\omega, x, u) \in M\} .
\end{aligned}
$$

We can show in a straightforward manner that for the set $\mathfrak{M}_{l}$ there exists the uniformity which is determined by the base $E(N, \epsilon), N, \epsilon>0$. It is easy to verify that the uniform space $\mathfrak{M}_{l}$ is metrizable and complete. Clearly $\mathfrak{M}_{c}$ is a closed subset of $\mathfrak{M}_{l}$. We consider the topological space $\mathfrak{M}_{c} \subset \mathfrak{M}_{l}$ which has the relative topology.

We consider the functionals of the form

$$
I^{(f)}(x, u)=\int_{\mathfrak{K}} f(\omega, x(\omega), u(\omega)) d \omega,
$$

where $(x, u) \in \Omega, f \in \mathfrak{M}_{\text {l }}$.

For each $f \in \mathfrak{M}_{l}$, we consider the problem of the absolute minimum

$$
I^{(f)}(x, u) \longrightarrow \min , \quad(x, u) \in \Omega,
$$

and set

$$
\mu(f)=\inf \left\{I^{(f)}(x, u):(x, u) \in \Omega\right\}
$$

It is easy to see that

$$
\mu(f)>-\infty \quad \text { for each } f \in \mathfrak{M}_{l}
$$

Define

$$
\mathfrak{M}_{l, \text { reg }}=\left\{f \in \mathfrak{M}_{l}: \mu(f)<\infty\right\}, \quad \mathfrak{M}_{c, \text { reg }}=\mathfrak{M}_{l, \text { reg }} \cap \mathfrak{M}_{c}
$$

Denote by $\overline{\mathfrak{M}}_{l \text {,reg }}$ the closure of $\mathfrak{M}_{l \text {,reg }}$ in $\mathfrak{M}_{l}$, and by $\overline{\mathfrak{M}}_{c \text {,reg }}$ the closure of $\mathfrak{M}_{c \text {,reg }}$ in $\mathfrak{M}_{c}$. The set $\mathfrak{M}_{l \text {,reg }}$ is an open subset of $\mathfrak{M}_{l}$, and a set $\mathfrak{M}_{c \text {,reg }}$ is an open subset of $\mathfrak{M}_{c}$ (see [22, Lemma 7.2]). We consider the topological subspaces $\overline{\mathfrak{M}}_{l, \text { reg }}, \overline{\mathfrak{M}}_{c \text {,reg }}$ which have the relative topology.

In [22] we established the following result which shows that generically the optimal control problem considered in this section has a unique solution. 
Theorem 3.1. There exist a set $\mathfrak{F}_{l} \subset \overline{\mathfrak{M}}_{l \text {,reg }}$ which is a countable intersection of open everywhere dense subsets of $\overline{\mathfrak{M}}_{l, \text { reg }}$, and a set $\mathfrak{F}_{c} \subset \overline{\mathfrak{M}}_{c, \text { reg }} \cap \mathfrak{F}_{l}$ which is a countable intersection of

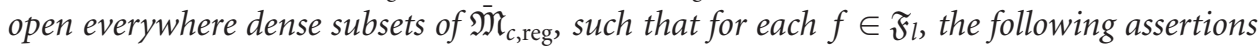
hold:

(1) $\mu(f)<\infty$ and there is a unique $\left(x^{(f)}, u^{(f)}\right) \in \Omega$ for which $I^{(f)}\left(x^{(f)}, u^{(f)}\right)=\mu(f)$,

(2) for each $\epsilon>0$, there exist a neighborhood $U$ of $f$ in $\mathfrak{M}_{l}$ and a number $\delta>0$ such that for each $g \in U$ and each $(x, u) \in \Omega$ satisfying $I^{(g)}(x, u) \leq \mu(g)+\delta$, the following relation holds:

$$
\operatorname{mes}\left\{\omega \in \mathfrak{K}:\left|x(\omega)-x^{(f)}(\omega)\right|+\left|u(\omega)-u^{(f)}(\omega)\right| \geq \epsilon\right\} \leq \epsilon .
$$

\section{Generic well-posedness in nonconvex optimal control}

We use the following notations and definitions. Let $k \geq 1$ be an integer. We again denote by $\operatorname{mes}(E)$ the Lebesgue measure of a measurable set $E \subset \mathbb{R}^{k}$ and by $|\cdot|$ the Euclidean norm in $\mathbb{R}^{k}$. Denote by $\langle\cdot, \cdot\rangle$ the scalar product in $\mathbb{R}^{k}$. We use the convention that $\infty-$ $\infty=0$. For any $f \in C^{q}\left(\mathbb{R}^{k}\right)$, we set

$$
\begin{aligned}
\|f\|_{C^{q}} & =\|f\|_{C^{q}\left(\mathbb{R}^{k}\right)} \\
& =\sup _{z \in \mathbb{R}^{k}}\left\{\left|\frac{\partial^{|\alpha|} f(z)}{\partial x_{1}^{\alpha_{1}} \cdots \partial x_{k}^{\alpha_{k}}}\right|: \alpha_{i} \geq 0 \text { is an integer, } i=1, \ldots, k,|\alpha| \leq q\right\},
\end{aligned}
$$

where $|\alpha|=\sum_{i=1}^{k} \alpha_{i}$

For each function $f: X \rightarrow[-\infty, \infty]$ where $X$ is nonempty, we set $\inf (f)=\inf \{f(x)$ : $x \in X\}$. For each set-valued mapping $U: X \rightarrow 2^{Y} \backslash\{\varnothing\}$ where $X$ and $Y$ are nonempty, we set

$$
\operatorname{graph}(U)=\{(x, y) \in X \times Y: y \in U(x)\}
$$

We consider topological spaces with two topologies where one is weaker than the other. (Note that they can coincide.) We refer to them as the weak and the strong topologies, respectively. If $(X, d)$ is a metric space with a metric $d$ and $Y \subset X$, then usually $Y$ is also endowed with the metric $d$ (unless another metric is introduced in $Y$ ). Assume that $X_{1}$ and $X_{2}$ are topological spaces and that each of them is endowed with a weak and a strong topology. Then for the product $X_{1} \times X_{2}$, we also introduce a pair of topologies: a weak topology which is the product of the weak topologies of $X_{1}$ and $X_{2}$ and a strong topology which is the product of the strong topologies of $X_{1}$ and $X_{2}$. If $Y \subset X_{1}$, then we consider the topological subspace $Y$ with the relative weak and strong topologies (unless other topologies are introduced). If $\left(X_{i}, d_{i}\right), i=1,2$, are metric spaces with the metrics $d_{1}$ and $d_{2}$, respectively, then the space $X_{1} \times X_{2}$ is endowed with the metric $d$ defined by

$$
d\left(\left(x_{1}, x_{2}\right),\left(y_{1}, y_{2}\right)\right)=d_{1}\left(x_{1}, y_{1}\right)+d_{2}\left(x_{2}, y_{2}\right), \quad\left(x_{i}, y_{i}\right) \in X \times Y, \quad i=1,2 .
$$

Let $m, n, N \geq 1$ be integers. We assume that $\Omega$ is a fixed bounded domain in $\mathbb{R}^{m}$, $H(t, x, u)$ is a fixed continuous function defined on $\Omega \times \mathbb{R}^{n} \times \mathbb{R}^{N}$ with values in $\mathbb{R}^{m n}$ such that $H(t, x, u)=\left(H_{i}\right)_{i=1}^{n}$ and $H_{i}=\left(H_{i j}\right)_{j=1}^{m}, i=1, \ldots, n, B_{1}$ and $B_{2}$ are fixed nonempty 
closed subsets of $\mathbb{R}^{n}$ and $\theta^{*}=\left(\theta_{i}^{*}\right)_{i=1}^{n} \in\left(W^{1,1}(\Omega)\right)^{n}$ is also fixed. Here

$$
W^{1,1}(\Omega)=\left\{u \in L^{1}(\Omega): \frac{\partial u}{\partial x_{j}} \in L^{1}(\Omega), j=1, \ldots, m\right\}
$$

and $W_{0}^{1,1}(\Omega)$ is the closure of $C_{0}^{\infty}(\Omega)$ in $W^{1,1}(\Omega)$, where $C_{0}^{\infty}(\Omega)$ is the space of smooth functions $u: \Omega \rightarrow \mathbb{R}^{1}$ with compact support in $\Omega$.

If $m=1$, then we assume that $\Omega=\left(T_{1}, T_{2}\right)$, where $T_{1}$ and $T_{2}$ are fixed real numbers for which $T_{1}<T_{2}$.

For a function $u=\left(u_{1}, \ldots, u_{n}\right)$, where $u_{i} \in W^{1,1}(\Omega), i=1, \ldots, n$, we set

$$
\nabla u_{i}=\left(\frac{\partial u_{i}}{\partial x_{j}}\right)_{j=1}^{m}, \quad i=1, \ldots, n, \nabla u=\left(\nabla u_{i}\right)_{i=1}^{n} .
$$

Define set-valued mappings $\tilde{A}: \Omega \rightarrow 2^{\mathbb{R}^{n}} \backslash\{\varnothing\}$ and $\tilde{U}: \Omega \times \mathbb{R}^{n} \rightarrow 2^{\mathbb{R}^{N}} \backslash\{\varnothing\}$ by

$$
\tilde{A}(t)=\mathbb{R}^{n}, \quad t \in \Omega, \quad \tilde{U}(t, x)=\mathbb{R}^{N}, \quad(t, x) \in \Omega \times \mathbb{R}^{n} .
$$

For each $A: \Omega \rightarrow 2^{\mathbb{R}^{n}} \backslash\{\varnothing\}$ and each $U: \operatorname{graph}(A) \rightarrow 2^{\mathbb{R}^{N}} \backslash\{\varnothing\}$ for which $\operatorname{graph}(U)$ is a closed subset of the space $\Omega \times \mathbb{R}^{n} \times \mathbb{R}^{N}$ with the product topology, we denote by $X(A, U)$ the set of all pairs of functions $(x, u)$, where $x=\left(x_{1}, \ldots, x_{n}\right) \in\left(W^{1,1}(\Omega)\right)^{n}, u=$ $\left(u_{1}, \ldots, u_{N}\right): \Omega \rightarrow \mathbb{R}^{N}$ is measurable and the following relations hold:

$$
\begin{gathered}
x(t) \in A(t), \quad t \in \Omega \text { almost everywhere (a.e.), } u(t) \in U(t, x(t)), \quad t \in \Omega \text { a.e., } \\
\quad \nabla x(t)=H(t, x(t), u(t)), \quad t \in \Omega \text { a.e., } \\
\text { if } m=1, \quad \text { then } x\left(T_{i}\right) \in B_{i}, i=1,2, \\
\text { if } m>1, \quad \text { then } x-\theta^{*} \in\left(W_{0}^{1,1}(\Omega)\right)^{n} .
\end{gathered}
$$

Note that in the definition of the space $X(A, U)$ we use the boundary condition $(4.7 \mathrm{c})$ in the case $m=1$ while in the case $m>1$ we use the boundary condition $(4.7 \mathrm{~d})$. Both of them are common in the literature. We do this to provide a unified treatment for both cases. Note that the main result of the section is valid in the case $m=1$ for a class of Bolza problems (with the same boundary condition (4.7c)) while in the case $m>1$ it holds for a class of Lagrange problems (with the same boundary condition $(4.7 \mathrm{~d})$ ).

To be more precise, we have to define elements of $X(A, U)$ as classes of pairs equivalent in the sense that $\left(x_{1}, u_{1}\right)$ and $\left(x_{2}, u_{2}\right)$ are equivalent if and only if $x_{2}(t)=x_{1}(t), u_{2}(t)=$ $u_{1}(t), t \in \Omega$ a.e. If $m=1$, then by an appropriate choice of representatives, $W^{1,1}\left(T_{1}, T_{2}\right)$ can be identified with the set of absolutely continuous functions $x:\left[T_{1}, T_{2}\right] \rightarrow \mathbb{R}^{1}$, and we will henceforth assume that this has been done.

Let $A: \Omega \rightarrow 2^{\mathbb{R}^{n}} \backslash\{\varnothing\}, U: \operatorname{graph}(A) \rightarrow 2^{\mathbb{R}^{N}} \backslash\{\varnothing\}$ and let graph $(U)$ be a closed subset of the space $\Omega \times \mathbb{R}^{n} \times \mathbb{R}^{N}$ with the product topology. 
For the set $X(A, U)$ defined above, we consider the uniformity which is determined by the following base:

$$
\begin{aligned}
E_{X}(\epsilon)=\{ & \left(\left(x_{1}, u_{1}\right),\left(x_{2}, u_{2}\right)\right) \in X(A, U) \times X(A, U): \\
& \left.\operatorname{mes}\left\{t \in \Omega:\left|x_{1}(t)-x_{2}(t)\right|+\left|u_{1}(t)-u_{2}(t)\right| \geq \epsilon\right\} \leq \epsilon\right\},
\end{aligned}
$$

where $\epsilon>0$. It is easy to see that the uniform space $X(A, U)$ is metrizable (by a metric $\rho$ ). In the space $X(A, U)$ we consider the topology induced by the metric $\rho$.

Next we define spaces of integrands associated with the maps $A$ and $U$. By $M(A, U)$ we denote the set of all functions $f: \operatorname{graph}(U) \rightarrow \mathbb{R}^{1} \cup\{\infty\}$ with the following properties:

(i) $f$ is measurable with respect to the $\sigma$-algebra generated by products of Lebesgue measurable subsets of $\Omega$ and Borel subsets of $\mathbb{R}^{n} \times \mathbb{R}^{N}$;

(ii) $f(t, \cdot, \cdot)$ is lower semicontinuous for a.e. $t \in \Omega$;

(iii) for each $\epsilon>0$, there exists an integrable scalar function $\psi_{\epsilon}(t) \geq 0, t \in \Omega$, such that $|H(t, x, u)| \leq \psi_{\epsilon}(t)+\epsilon f(t, x, u)$ for all $(t, x, u) \in \operatorname{graph}(U)$.

Due to the property (i) for every $f \in \mathcal{M}(A, U)$ and every $(x, u) \in X(A, U)$, the function $f(t, x(t), u(t)), t \in \Omega$, is measurable.

Denote by $\mathcal{M}^{l}(A, U)$ (resp., $\left.\mathcal{M}^{c}(A, U)\right)$ the set of all lower semicontinuous (resp., finitevalued continuous) functions $f: \operatorname{graph}(U) \rightarrow \mathbb{R}^{1} \cup\{\infty\}$ in $\mathcal{M}(A, U)$. Now we equip the set $M(A, U)$ with the strong and weak topologies. For the space $M(A, U)$, we consider the uniformity determined by the following base:

$$
\begin{aligned}
E_{\mathcal{M}}(\epsilon)=\{ & (f, g) \in \mathcal{M}(A, U) \times M(A, U): \\
& |f(t, x, u)-g(t, x, u)| \leq \epsilon,(t, x, u) \in \operatorname{graph}(U)\},
\end{aligned}
$$

where $\epsilon>0$. It is easy to see that the uniform space $M(A, U)$ with this uniformity is metrizable (by a metric $d_{\mathcal{M}}$ ) and complete. This uniformity generates in $\mathcal{M}(A, U)$ the strong topology. Clearly $\mathcal{M}^{l}(A, U)$ and $\mathcal{M}^{c}(A, U)$ are closed subsets of $\mathcal{M}(A, U)$ with this topology.

For each $\epsilon>0$, we set

$$
\begin{aligned}
E_{M_{w}}(\epsilon)=\{ & (f, g) \in \mathcal{M}(A, U) \times M(A, U): \text { there exists a nonnegative } \\
& \phi \in L^{1}(\Omega) \text { such that } \int_{\Omega} \phi(t) d t \leq 1, \text { and for a.e. } t \in \Omega, \\
& |f(t, x, u)-g(t, x, u)|<\epsilon+\epsilon \max \{|f(t, x, u)|,|g(t, x, u)|\}+\epsilon \phi(t) \\
& \text { for each } x \in A(t), \text { each } u \in U(t, x)\} .
\end{aligned}
$$

Using the following simple lemma, we can easily show that for the set $M(A, U)$ there exists the uniformity which is determined by the base $E_{\mu_{w}}(\epsilon), \epsilon>0$. This uniformity induces in $M(A, U)$ the weak topology.

Lemma 4.1. Let $a, b \in \mathbb{R}^{1}, \epsilon \in(0,1), \Delta \geq 0$, and

$$
|a-b|<(1+\Delta) \epsilon+\epsilon \max \{|a|,|b|\} .
$$


Then

$$
|a-b|<(1+\Delta)\left(\epsilon+\epsilon^{2}(1-\epsilon)^{-1}\right)+\epsilon(1-\epsilon)^{-1} \min \{|a|,|b|\} .
$$

Denote by $C_{l}\left(B_{1} \times B_{2}\right)$ the set of all lower semicontinuous functions $\xi: B_{1} \times B_{2} \rightarrow$ $\mathbb{R}^{1} \cup\{\infty\}$ bounded from below. We also equip the set $C_{l}\left(B_{1} \times B_{2}\right)$ with strong and weak topologies. For the set $C_{l}\left(B_{1} \times B_{2}\right)$, we consider the uniformity determined by the following base:

$$
E_{c}(\epsilon)=\left\{(\xi, h) \in C_{l}\left(B_{1} \times B_{2}\right) \times C_{l}\left(B_{1} \times B_{2}\right):|\xi(z)-h(z)| \leq \epsilon, z \in B_{1} \times B_{2}\right\},
$$

where $\epsilon>0$. It is easy to see that the uniform space $C_{l}\left(B_{1} \times B_{2}\right)$ is metrizable (by a metric $d_{c}$ ) and complete. This metric induces in $C_{l}\left(B_{1} \times B_{2}\right)$ the strong topology.

For any $\epsilon>0$, we set

$$
\begin{aligned}
& E_{c w}(\epsilon)=\left\{(\xi, h) \in C_{l}\left(B_{1} \times B_{2}\right) \times C_{l}\left(B_{1} \times B_{2}\right):\right. \\
&\left.|\xi(z)-h(z)|<\epsilon+\epsilon \max \{|\xi(z)|,|h(z)|\}, z \in B_{1} \times B_{2}\right\},
\end{aligned}
$$

where $\epsilon>0$. By using Lemma 4.1 , we can easily show that for the set $C_{l}\left(B_{1} \times B_{2}\right)$ there exists a uniformity which is determined by the base $E_{c w}(\epsilon), \epsilon>0$. This uniformity induces in $C_{l}\left(B_{1} \times B_{2}\right)$ the weak topology. Denote by $C\left(B_{1} \times B_{2}\right)$ the set of all finite-valued continuous functions $h$ in $C_{l}\left(B_{1} \times B_{2}\right)$. Clearly it is a closed subset of $C_{l}\left(B_{1} \times B_{2}\right)$ with the weak topology.

In the case $m>1$ for each $f \in M(A, U)$ we define $I^{(f)}: X(A, U) \rightarrow \mathbb{R}^{1} \cup\{\infty\}$ by

$$
I^{(f)}(x, u)=\int_{\Omega} f(t, x(t), u(t)) d t, \quad(x, u) \in X(A, U) .
$$

In the case $m=1$ for each $f \in \mathcal{M}(A, U)$ and each $\xi \in C_{l}\left(B_{1} \times B_{2}\right)$ we define $I^{(f, \xi)}: X(A, U) \rightarrow$ $\mathbb{R}^{1} \cup\{\infty\}$ by

$$
I^{(f, \xi)}(x, u)=\int_{T_{1}}^{T_{2}} f(t, x(t), u(t)) d t+\xi\left(x\left(T_{1}\right), x\left(T_{2}\right)\right), \quad(x, u) \in X(A, U) .
$$

We showed (see [20, Propositions 4.1 and 4.2]) that in both cases (4.15) and (4.16) define lower semicontinuous functionals on $X(A, U)$.

From now on in this section, we consider a fixed set-valued mapping $A: \Omega \rightarrow 2^{\mathbb{R}^{n}}$ । $\{\varnothing\}$ for which graph $(A)$ is a closed subset of the space $\Omega \times \mathbb{R}^{n}$ with the product topology. Denote by $\tilde{U}_{A}$ the restriction of $\tilde{U}$ (see (4.6)) to the graph $(A)$. Namely,

$$
\tilde{U}_{A}: \operatorname{graph}(A) \longrightarrow 2^{\mathbb{R}^{N}}, \quad \tilde{U}_{A}(t, x)=\mathbb{R}^{N}, \quad(t, x) \in \operatorname{graph}(A) .
$$

We consider functionals $I^{(f, \xi)}$ with $(f, \xi) \in \mathcal{M}\left(A, \tilde{U}_{A}\right) \times C_{l}\left(B_{1} \times B_{2}\right)$ (in the case $m=1$ ) and functionals $I^{(f)}$ with $f \in M\left(A, \tilde{U}_{A}\right)$ (in the case $m>1$ ) defined on the space $X\left(A, \tilde{U}_{A}\right)$ (see (4.7)). The main result of this section is established for several classes of optimal control problems with different corresponding spaces of the integrands which are subsets of the space $M\left(A, \tilde{U}_{A}\right)$. The subspaces of lower semicontinuous and continuous integrands $\left(M^{l}\left(A, \tilde{U}_{A}\right)\right.$ and $\left.\mathcal{M}^{c}\left(A, \tilde{U}_{A}\right)\right)$ have already been defined. Now we define subspaces 
of $\mathcal{M}\left(A, \tilde{U}_{A}\right)$ which consist of integrands differentiable with respect to the control variable $u$.

Let $k \geq 1$ be an integer. Denote by $\mathcal{M}_{k}\left(A, \widetilde{U}_{A}\right)$ the set of all finite-valued $f \in M\left(A, \widetilde{U}_{A}\right)$ such that for each $(t, x) \in \operatorname{graph}(A)$ the function $f(t, x, \cdot) \in C^{k}\left(\mathbb{R}^{N}\right)$. We consider the topological subspace $M_{k}\left(A, \tilde{U}_{A}\right) \subset \mathcal{M}\left(A, \tilde{U}_{A}\right)$ with the relative weak topology. The strong topology on $M_{k}\left(A, \tilde{U}_{A}\right)$ is induced by the uniformity which is determined by the following base:

$$
\begin{aligned}
E_{\mathcal{M}}(\epsilon)= & \left\{(f, g) \in \mathcal{M}_{k}\left(A, \tilde{U}_{A}\right) \times M_{k}\left(A, \tilde{U}_{A}\right):|f(t, x, u)-g(t, x, u)| \leq \epsilon \forall(t, x, u)\right. \\
& \left.\in \operatorname{graph}(A) \times \mathbb{R}^{N} \text { and }\|f(t, x, \cdot)-g(t, x, \cdot)\|_{C^{k}\left(\mathbb{R}^{N}\right)} \leq \epsilon \forall(t, x) \in \operatorname{graph}(A)\right\},
\end{aligned}
$$

where $\epsilon>0$. It is easy to see that the space $M_{k}\left(A, \tilde{U}_{A}\right)$ with this uniformity is metrizable (by a metric $d_{\mu, k}$ ) and complete. Define

$$
\mu_{k}^{l}\left(A, \tilde{U}_{A}\right)=M_{k}\left(A, \tilde{U}_{A}\right) \cap M^{l}\left(A, \tilde{U}_{A}\right), \quad M_{k}^{c}\left(A, \tilde{U}_{A}\right)=M_{k}\left(A, \tilde{U}_{A}\right) \cap M^{c}\left(A, \tilde{U}_{A}\right) .
$$

Clearly $\mathcal{M}_{k}^{l}\left(A, \tilde{U}_{A}\right)$ and $\mathcal{M}_{k}^{c}\left(A, \tilde{U}_{A}\right)$ are closed sets in $\mathcal{M}_{k}\left(A, \tilde{U}_{A}\right)$ with the strong topology.

Finally we define subspaces of $\mathcal{M}(\tilde{A}, \tilde{U})$ which consist of integrands differentiable with respect to the state variable $x$ and the control variable $u$. Denote by $\mathcal{M}_{k}^{*}(\tilde{A}, \tilde{U})$ the set of all $f: \Omega \times \mathbb{R}^{n} \times \mathbb{R}^{N} \rightarrow \mathbb{R}^{1}$ in $\mathcal{M}(\tilde{A}, \tilde{U})$ (see (4.6)) such that for each $t \in \Omega$ the function $f(t, \cdot, \cdot) \in C^{k}\left(\mathbb{R}^{n} \times \mathbb{R}^{N}\right)$. We consider the topological subspace $\mathcal{M}_{k}^{*}(\tilde{A}, \tilde{U}) \subset \mathcal{M}(\tilde{A}, \tilde{U})$ with the relative weak topology. The strong topology in $\mathcal{M}_{k}^{*}(\tilde{A}, \tilde{U})$ is induced by the uniformity which is determined by the following base:

$$
\begin{gathered}
E_{\mathcal{M}}^{*}(\epsilon)=\left\{(f, g) \in \mathcal{M}_{k}^{*}(\tilde{A}, \tilde{U}) \times M_{k}^{*}(\tilde{A}, \tilde{U}):|f(t, x, u)-g(t, x, u)| \leq \epsilon \forall(t, x, u)\right. \\
\left.\in \Omega \times \mathbb{R}^{n} \times \mathbb{R}^{N} \text { and } \| f(t, \cdot, \cdot)-\left.g(t, \cdot, \cdot)\right|_{C^{k}\left(\mathbb{R}^{n+N}\right)} \leq \epsilon \forall t \in \Omega\right\},
\end{gathered}
$$

where $\epsilon>0$. It is easy to see that the space $M_{k}^{*}(\tilde{A}, \tilde{U})$ with this uniformity is metrizable (by a metric $d_{M, k}^{*}$ ) and complete. Define

$$
\mu_{k}^{* l}(\tilde{A}, \tilde{U})=M_{k}^{*}(\tilde{A}, \tilde{U}) \cap M^{l}(\tilde{A}, \tilde{U}), \quad \mu_{k}^{* c}(\tilde{A}, \tilde{U})=M_{k}^{*}(\tilde{A}, \tilde{U}) \cap M^{c}(\tilde{A}, \tilde{U}) .
$$

Clearly $M_{k}^{* l}(\tilde{A}, \tilde{U})$ and $M_{k}^{* c}(\tilde{A}, \tilde{U})$ are closed sets in $M_{k}^{*}(\tilde{A}, \tilde{U})$ with the strong topology.

Thus we have defined all the spaces of integrands for which we will state our main result of this section. Now we will define a space of constraint maps $\mathscr{P}_{A}$. Denote by $S\left(\mathbb{R}^{N}\right)$ the set of all nonempty convex closed subsets of $\mathbb{R}^{N}$. For each $x \in \mathbb{R}^{N}$ and each $E \subset \mathbb{R}^{N}$, set $d_{H}(x, E)=\inf _{y \in E}|x-y|$. For each pair of sets $C_{1}, C_{2} \subset \mathbb{R}^{N}$,

$$
d_{H}\left(C_{1}, C_{2}\right)=\max \left\{\sup _{y \in C_{1}} d_{H}\left(y, C_{2}\right), \sup _{x \in C_{2}} d_{H}\left(x, C_{1}\right)\right\}
$$

is the Hausdorff distance between $C_{1}$ and $C_{2}$. For the space $S\left(\mathbb{R}^{N}\right)$, we consider the uniformity determined by the following base:

$$
E_{\mathbb{R}^{N}}(\epsilon)=\left\{\left(C_{1}, C_{2}\right) \in S\left(\mathbb{R}^{N}\right) \times S\left(\mathbb{R}^{N}\right): d_{H}\left(C_{1}, C_{2}\right) \leq \epsilon\right\}
$$


where $\epsilon>0$. It is well known that the space $S\left(\mathbb{R}^{N}\right)$ with this uniformity is metrizable and complete. Denote by $\mathscr{P}_{A}$ the set of all set-valued mappings $U: \operatorname{graph}(A) \rightarrow S\left(\mathbb{R}^{N}\right)$ such that $\operatorname{graph}(U)$ is a closed subset of the $\operatorname{space} \operatorname{graph}(A) \times \mathbb{R}^{N}$ with the product topology. For the space $\mathscr{P}_{A}$, we consider the uniformity determined by the following base:

$$
E_{\mathscr{P}_{A}}(\epsilon)=\left\{\left(U_{1}, U_{2}\right) \in \mathscr{P}_{A} \times \mathscr{P}_{A}: d_{H}\left(U_{1}(t, x), U_{2}(t, x)\right) \leq \epsilon \forall(t, x) \in \operatorname{graph}(A)\right\},
$$

where $\epsilon>0$. It is easy to see that the space $\mathscr{P}_{A}$ with this uniformity is metrizable and complete.

We consider the space $X\left(A, \tilde{U}_{A}\right)$ with the metric $\rho$ (see (4.8)). For each $U \in \mathscr{P}_{A}$, define

$$
S_{U}=X(A, U)=\left\{(x, u) \in X\left(A, \tilde{U}_{A}\right): u(t) \in U(t, x(t)), t \in \Omega \text { a.e. }\right\} .
$$

In the case $m=1$ for each $U \in \mathscr{P}_{A}$ and each $(f, \xi) \in M\left(A, \tilde{U}_{A}\right) \times C_{l}\left(B_{1} \times B_{2}\right)$ we consider the optimal control problem

$$
I^{(f, \xi)}(x, u) \longrightarrow \min , \quad(x, u) \in X(A, U)
$$

and in the case $m>1$ for each $U \in \mathscr{P}_{A}$ and each $f \in \mathcal{M}\left(A, \tilde{U}_{A}\right)$ we consider the optimal control problem

$$
I^{(f)}(x, u) \longrightarrow \min , \quad(x, u) \in X(A, U)
$$

We will state the main result of this section, Theorem 4.2, in such a manner that it will be applicable to the Bolza problem in case $m=1$ and to the Lagrange problem in case $m>1$, and also applicable for all the spaces of integrands defined above.

To meet this goal, we set $\mathscr{A}_{2}=\mathscr{P}_{A}$ and define a space $\mathscr{A}_{1}$ as follows:

$$
\mathscr{A}_{1}=\mathscr{A}_{11} \times \mathscr{A}_{12} \quad \text { if } m=1, \quad \mathscr{A}_{1}=\mathscr{A}_{11} \quad \text { if } m>1,
$$

where $\mathscr{A}_{12}$ is either $C_{l}\left(B_{1} \times B_{2}\right)$ or $C\left(B_{1} \times B_{2}\right)$ or a singleton $\{\xi\} \subset C_{l}\left(B_{1} \times B_{2}\right)$, and $\mathscr{A}_{11}$ is one of the following spaces:

$$
\begin{gathered}
\mathcal{M}\left(A, \tilde{U}_{A}\right), \quad M^{l}\left(A, \tilde{U}_{A}\right), \quad M^{c}\left(A, \tilde{U}_{A}\right), \\
\mathcal{M}_{k}\left(A, \tilde{U}_{A}\right), \quad M_{k}^{l}\left(A, \tilde{U}_{A}\right), \quad M_{k}^{c}\left(A, \tilde{U}_{A}\right) \quad \text { (here } k \geq 1 \text { is an integer), } \\
\left.\mathcal{M}_{k}^{*}(\tilde{A}, \tilde{U}), \quad M_{k}^{* l}(\tilde{A}, \tilde{U}), \quad M_{k}^{* c}(\tilde{A}, \tilde{U}) \quad \text { (here } k \geq 1 \text { is an integer and } A=\tilde{A}\right) .
\end{gathered}
$$

For each $a=\left(a_{1}, a_{2}\right) \in \mathscr{A}_{1} \times \mathscr{A}_{2}$, we define $J_{a}: X\left(A, \tilde{U}_{A}\right) \rightarrow \mathbb{R}^{1} \cup\{\infty\}$ by

$$
J_{a}(x, u)=I^{\left(a_{1}\right)}(x, u), \quad(x, u) \in S_{a_{2}}, \quad J_{a}(x, u)=\infty, \quad(x, u) \in X\left(A, \tilde{U}_{A}\right) \backslash S_{a_{2}} .
$$

In [20], we showed that $J_{a}$ is lower semicontinuous for all $a \in \mathscr{A}_{1} \times A_{2}$. Denote by $\mathscr{A}$ the closure of the set $\left\{a \in \mathscr{A}_{1} \times \mathscr{A}_{2}: \inf \left(J_{a}\right)<\infty\right\}$ in the space $\mathscr{A}_{1} \times A_{2}$ with the strong topology. We assume that $\mathscr{A}$ is nonempty. The following theorem established in [20] is the main result of this section. 
Theorem 4.2. There exists an everywhere dense (in the strong topology) set $\mathscr{B} \subset \mathscr{A}$ which is a countable intersection of open (in the weak topology) subsets of $\mathscr{A}$ such that for any a $\in \mathscr{B}$, the following assertions hold:

(1) $\inf \left(J_{a}\right)$ is finite and attained at a unique pair $(\bar{x}, \bar{u}) \in X\left(A, \tilde{U}_{A}\right)$,

(2) for each $\epsilon>0$ there are a neighborhood $\mathscr{V}$ of $a$ in $\mathscr{A}$ with the weak topology and $\delta>0$ such that for each $b \in \mathscr{V}, \inf \left(J_{b}\right)$ is finite and if $(z, w) \in X\left(A, \tilde{U}_{A}\right)$ satisfies $J_{b}(z, w) \leq \inf \left(J_{b}\right)+\delta$, then $\rho((\bar{x}, \bar{u}),(z, w)) \leq \epsilon$ and $\left|J_{b}(z, w)-J_{a}(\bar{x}, \bar{u})\right| \leq \epsilon$.

\section{Generic variational principle}

Theorem 4.2 is obtained as a realization of a variational principle which was introduced in [20]. This variational principle is a modification of the variational principle in [10].

We consider a metric space $(X, \rho)$ which is called the domain space and a complete metric space $(\mathscr{A}, d)$ which is called the data space. We always consider the set $X$ with the topology generated by the metric $\rho$. For the space $\mathscr{A}$, we consider the topology generated by the metric $d$. This topology will be called the strong topology. In addition to the strong topology, we also consider a weaker topology on $\mathscr{A}$ which is not necessarily Hausdorff. This topology will be called the weak topology. (Note that these topologies can coincide.) We assume that with every $a \in \mathscr{A}$ a lower semicontinuous function $f_{a}$ on $X$ is associated with values in $\mathbb{R}=[-\infty, \infty]$. In our study, we use the following basic hypotheses about the functions.

(H1) For any $a \in \mathscr{A}$, any $\epsilon>0$, and any $\gamma>0$, there exist a nonempty open set $\mathcal{W}$ in $\mathscr{A}$ with the weak topology, $x \in X, \alpha \in \mathbb{R}^{1}$, and $\eta>0$ such that

$$
\mathscr{W} \cap\{b \in \mathscr{A}: d(a, b)<\epsilon\} \neq \varnothing
$$

and for any $b \in \mathcal{W}$,

(i) $\inf \left(f_{b}\right)$ is finite;

(ii) if $z \in X$ is such that $f_{b}(z) \leq \inf \left(f_{b}\right)+\eta$, then $\rho(z, x) \leq \gamma$ and $\left|f_{b}(z)-\alpha\right| \leq \gamma$.

(H2) If $a \in \mathscr{A}, \inf \left(f_{a}\right)$ is finite, $\left\{x_{n}\right\}_{n=1}^{\infty} \subset X$ is a Cauchy sequence, and the sequence $\left\{f_{a}\left(x_{n}\right)\right\}_{n=1}^{\infty}$ is bounded, then the sequence $\left\{x_{n}\right\}_{n=1}^{\infty}$ converges in $X$.

In [20] we showed (see Theorem 5.1 below) that if (H1) and (H2) hold, then for a generic $a \in \mathscr{A}$ the minimization problem $f_{a}(x) \rightarrow \min , x \in X$, has a unique solution. This result generalizes the variational principle in [10, Theorem 2.2] which was obtained for the complete domain space $(X, \rho)$. Note that if $(X, \rho)$ is complete, the weak and strong topologies on $\mathscr{A}$ coincide and for any $a \in \mathscr{A}$ the function $f_{a}$ is not identically $\infty$, then the variational principles in [10] and in this section are equivalent.

For the classes of optimal control problems considered in this paper, the domain space is usually the space $X\left(A, \tilde{U}_{A}\right)$ with the metric $\rho$ (see (4.8)) which is not complete. Since the variational principle in [10] was established only for complete domain spaces, it cannot be applied to these classes of optimal control problems. Fortunately, instead of the completeness assumption, we can use (H2) and this hypothesis holds for spaces of integrands (integrand-map pairs) which satisfy the Cesari growth condition. In [20] we established the following result. 
Theorem 5.1. Assume that (H1) and (H2) hold. Then there exists an everywhere dense (in the strong topology) set $\mathscr{B} \subset \mathscr{A}$ which is a countable intersection of open (in the weak topology) subsets of $\mathscr{A}$ such that for any $a \in \mathscr{B}$, the following assertions hold:

(1) $\inf \left(f_{a}\right)$ is finite and attained at a unique point $\bar{x} \in X$,

(2) for each $\epsilon>0$, there are a neighborhood $\mathscr{V}$ of a in $\mathcal{A}$ with the weak topology and $\delta>0$ such that for each $b \in \mathscr{V}, \inf \left(f_{b}\right)$ is finite and if $z \in X$ satisfies $f_{b}(z) \leq \inf \left(f_{b}\right)+\delta$, then $\rho(\bar{x}, z) \leq \epsilon$ and $\left|f_{b}(z)-f_{a}(\bar{x})\right| \leq \epsilon$.

Following the tradition, we can summarize the theorem by saying that under the assumptions $(\mathrm{H} 1)$ and $(\mathrm{H} 2)$ the minimization problem for $f_{a}$ on $(X, \rho)$ is generically strongly well-posed with respect to $\mathscr{A}$.

The proof of Theorem 4.2 consists in verifying that hypotheses (H1) and (H2) hold for the space of integrand-map pairs introduced in Section 4. To simplify the verification of (H1) in [20] we introduced new assumptions (A1)-(A4) and showed that they imply (H1) (see Proposition 5.3 below).

Let $(X, \rho)$ be a metric space with the topology generated by the metric $\rho$ and let $\left(\mathscr{A}_{1}, d_{1}\right),\left(\mathscr{A}_{2}, d_{2}\right)$ be metric spaces. For the space $\mathscr{A}_{i}(i=1,2)$, we consider the topology generated by the metric $d_{i}$. This topology is called the strong topology. In addition to the strong topology we consider a weak topology on $\mathscr{A}_{i}, i=1,2$.

Assume that with every $a \in \mathscr{A}_{1}$ a lower semicontinuous function $\phi_{a}: X \rightarrow \mathbb{R}^{1} \cup\{\infty\}$ is associated and with every $a \in \mathscr{A}_{2}$ a set $S_{a} \subset X$ is associated. For each $a=\left(a_{1}, a_{2}\right) \in$ $\mathscr{A}_{1} \times \mathscr{A}_{2}$, define $f_{a}: X \rightarrow \mathbb{R}^{1} \cup\{\infty\}$ by

$$
f_{a}(x)=\phi_{a_{1}}(x) \quad \forall x \in S_{a_{2}}, \quad f_{a}(x)=\infty \quad \forall x \in X \backslash S_{a_{2}} .
$$

Denote by $\mathscr{A}$ the closure of the set $\left\{a \in \mathscr{A}_{1} \times \mathscr{A}_{2}: \inf \left(f_{a}\right)<\infty\right\}$ in the space $\mathscr{A}_{1} \times \mathscr{A}_{2}$ with the strong topology. We assume that $\mathscr{A}$ is nonempty.

We use the following hypotheses.

(A1) For each $a_{1} \in \mathscr{A}_{1}, \inf \left(\phi_{a_{1}}\right)>-\infty$ and for each $a \in \mathscr{A}_{1} \times \mathscr{A}_{2}$, the function $f_{a}$ is lower semicontinuous.

(A2) For each $a \in \mathscr{A}_{1}$ and each $D, \epsilon>0$, there is a neighborhood $U$ of $a$ in $\mathscr{A}_{1}$ with the weak topology such that for each $b \in \mathcal{U}$ and each $x \in X$ satisfying $\min \left\{\phi_{a}(x)\right.$, $\left.\phi_{b}(x)\right\} \leq D$, the relation $\left|\phi_{a}(x)-\phi_{b}(x)\right| \leq \epsilon$ holds.

(A3) For each $\gamma \in(0,1)$, there exist positive numbers $\epsilon(\gamma)$ and $\delta(\gamma)$ such that $\epsilon(\gamma)$, $\delta(\gamma) \rightarrow 0$ as $\gamma \rightarrow 0$ and the following property holds.

For each $\gamma \in(0,1)$, each $a \in \mathscr{A}_{1}$, each nonempty set $Y \subset X$, and each $\bar{x} \in Y$ for which

$$
\phi_{a}(\bar{x}) \leq \inf \left\{\phi_{a}(z): z \in Y\right\}+\delta(\gamma)<\infty,
$$

there is $\bar{a} \in \mathscr{A}_{1}$ such that the following conditions hold:

$$
d_{1}(a, \bar{a}) \leq \epsilon(\gamma), \quad \phi_{\bar{a}}(z) \geq \phi_{a}(z), \quad z \in X, \quad \phi_{\bar{a}}(\bar{x}) \leq \phi_{a}(\bar{x})+\delta(\gamma) ;
$$

for each $y \in Y$ satisfying

$$
\phi_{\bar{a}}(y) \leq \inf \left\{\phi_{\bar{a}}(z): z \in Y\right\}+2 \delta(\gamma)
$$

the inequality $\rho(y, \bar{x}) \leq \gamma$ is valid. 
(A4) For each $a=\left(a_{1}, a_{2}\right) \in \mathscr{A}_{1} \times \mathscr{A}_{2}$ satisfying $\inf \left(f_{a}\right)<\infty$ and each $\epsilon, \delta>0$, there exist $\bar{a}_{2} \in \mathscr{A}_{2}, \bar{x} \in S_{\bar{a}_{2}}$, and an open set $\mathcal{U}$ in $\mathscr{A}_{2}$ with the weak topology such that

$$
\begin{gathered}
d_{2}\left(a_{2}, \bar{a}_{2}\right)<\epsilon, \quad U \cap\left\{b \in \mathscr{A}_{2}: d_{2}\left(b, a_{2}\right)<\epsilon\right\} \neq \varnothing, \\
\phi_{a_{1}}(\bar{x}) \leq \inf \left\{\phi_{a_{1}}(z): z \in S_{\bar{a}_{2}}\right\}+\delta<\infty, \\
\bar{x} \in S_{b} \subset S_{\bar{a}_{2}} \quad \forall b \in \mathcal{U} .
\end{gathered}
$$

Remark 5.2. Assume that (A3) holds. In [20] we showed that the numbers $\epsilon(\gamma)$ and $\delta(\gamma)$ can be chosen such that $0<\delta(\gamma) \leq \epsilon(\gamma) \leq \gamma$.

The following result was established in [20].

Proposition 5.3. Assume that (A1)-(A4) hold. Then (H1) holds for the space $\mathscr{A}$.

Remark 5.4. In the proof of Proposition 5.3, (see [20, Proposition 3.1]) for any $a=\left(a_{1}\right.$, $\left.a_{2}\right) \in \mathscr{A}_{1} \times \mathscr{A}_{2}$ satisfying $\inf \left(f_{a}\right)<\infty$ and any $\epsilon>0$, we constructed an open set $\mathscr{V}$ in $\mathscr{A}_{1}$ with the weak topology and an open set $\mathcal{U}$ in $\mathscr{A}_{2}$ with the weak topology which satisfy

$$
\mathscr{V} \cap\left\{b \in \mathscr{A}_{1}: d_{1}\left(b, a_{1}\right)<\epsilon\right\} \neq \varnothing, \quad \mathcal{U} \cap\left\{b \in \mathscr{A}_{2}: d_{2}\left(b, a_{2}\right)<\epsilon\right\} \neq \varnothing
$$

and such that $\inf \left(f_{b}\right)<\infty$ for each $b=\left(b_{1}, b_{2}\right) \in \mathscr{V} \times \mathscr{U}$. This implies that there exists an open set $\mathscr{F}$ in $\mathscr{A}_{1} \times \mathscr{A}_{2}$ with the weak topology such that $\inf \left(f_{a}\right)<\infty$ for all $a \in \mathscr{F}$ and $\mathscr{A}$ is the closure of $\mathscr{F}$ in the space $\mathscr{A}_{1} \times \mathscr{A}_{2}$ with the strong topology.

\section{Preliminary results for hypotheses (A2) and (H2)}

In this section, we present several auxiliary results obtained in [20].

Assume that $A: \Omega \rightarrow 2^{\mathbb{R}^{n}} \backslash\{\varnothing\}, U: \operatorname{graph}(A) \rightarrow 2^{\mathbb{R}^{N}} \backslash\{\varnothing\}$ and that $\operatorname{graph}(U)$ is a closed subset of the space $\Omega \times \mathbb{R}^{n} \times \mathbb{R}^{N}$ with the product topology. Consider the spaces $X(A, U), \mathcal{M}(A, U)$, and $C_{l}\left(B_{1} \times B_{2}\right)$ introduced in Section 4 .

Proposition 6.1. Let $f \in M(A, U),(x, u) \in X(A, U),\left\{\left(x_{i}, u_{i}\right)\right\}_{i=1}^{\infty} \subset X(A, U)$, and let $\rho\left(\left(x_{i}, u_{i}\right),(x, u)\right) \rightarrow 0$ as $i \rightarrow \infty$. Then

$$
\int_{\Omega} f(t, x(t), u(t)) d t \leq \liminf _{i \rightarrow \infty} \int_{\Omega} f\left(t, x_{i}(t), u_{i}(t)\right) d t
$$

The following proposition is an auxiliary result for hypothesis (H2).

Proposition 6.2. Assume that $f \in M(A, U),\left\{\left(x_{i}, u_{i}\right)\right\}_{i=1}^{\infty} \subset X(A, U)$ is a Cauchy sequence, and the sequence $\left\{\int_{\Omega} f\left(t, x_{i}(t), u_{i}(t)\right) d t\right\}_{i=1}^{\infty}$ is bounded. Then there is $\left(x_{*}, u_{*}\right) \in X(A, U)$ such that $\left(x_{i}, u_{i}\right)$ converges to $\left(x_{*}, u_{*}\right)$ as $i \rightarrow \infty$ in $X(A, U)$ and, moreover, if $m=1$, then $x_{i}(t) \rightarrow x_{*}(t)$ as $i \rightarrow \infty$ uniformly on $\left[T_{1}, T_{2}\right]$.

Proposition 6.3. Let $h \in C_{l}\left(B_{1} \times B_{2}\right)$ and $\epsilon, D>0$. Then there exists a neighborhood $\mathscr{V}$ of $h$ in $C_{l}\left(B_{1} \times B_{2}\right)$ with the weak topology such that for each $\xi \in \mathscr{V}$ and each $x \in B_{1} \times B_{2}$ which satisfies $\min \{\xi(x), h(x)\} \leq D$, the relation $|\xi(x)-h(x)| \leq \epsilon$ holds. 
Corollary 6.4. Let $h \in C_{l}\left(B_{1} \times B_{2}\right)$ and $\epsilon>0$. Then there is a neighborhood $\mathscr{V}$ of $h$ in $C_{l}\left(B_{1} \times B_{2}\right)$ with the weak topology such that for each $\xi \in \mathscr{V}$, the inequality $|\inf (\xi)-\inf (h)|$ $\leq \epsilon$ holds.

The following proposition is an auxiliary result for assumption (A2).

Proposition 6.5. Let $f \in M(A, U)$ and $\epsilon \in(0,1), D>0$. Then there exists a neighborhood $\mathscr{V}$ of $f$ in $M(A, U)$ with the weak topology such that for each $g \in \mathscr{V}$ and each $(x, u) \in$ $X(A, U)$ satisfying

$$
\min \left\{\int_{\Omega} f(t, x(t), u(t)) d t, \int_{\Omega} g(t, x(t), u(t)) d t\right\} \leq D
$$

the following relation holds:

$$
\left|\int_{\Omega} f(t, x(t), u(t)) d t-\int_{\Omega} g(t, x(t), u(t)) d t\right| \leq \epsilon .
$$

Corollary 6.6. Let $f \in M(A, U)$ and $\epsilon>0$. Then there exists a neighborhood $\mathscr{V}$ of $f$ in $M(A, U)$ with the weak topology such that for all $g \in \mathscr{V}$,

$$
\begin{aligned}
\mid \inf \{ & \left.\int_{\Omega} f(t, x(t), u(t)) d t:(x, u) \in X(A, U)\right\} \\
& -\inf \left\{\int_{\Omega} g(t, x(t), u(t)) d t:(x, u) \in X(A, U)\right\} \mid<\epsilon .
\end{aligned}
$$

Proposition 6.7. Let $m=1, f \in M(A, U), h \in C_{l}\left(B_{1} \times B_{2}\right)$, and $\epsilon \in(0,1), D>0$. Then there exist a neighborhood $U$ of $f$ in $M(A, U)$ with the weak topology and a neighborhood $\mathcal{V}$ of $h$ in $C_{l}\left(B_{1} \times B_{2}\right)$ with the weak topology such that for each $(\xi, g) \in \mathscr{V} \times \mathscr{U}$ and each $(x, u) \in X(A, U)$ which satisfies

$$
\min \left\{I^{(f, h)}(x, u), I^{(g, \xi)}(x, u)\right\} \leq D,
$$

the following relations are valid:

$$
\begin{gathered}
\left|h\left(x\left(T_{1}\right), x\left(T_{2}\right)\right)-\xi\left(x\left(T_{1}\right), x\left(T_{2}\right)\right)\right| \leq \epsilon, \\
\left|\int_{T_{1}}^{T_{2}}[f(t, x(t), u(t))-g(t, x(t), u(t))] d t\right| \leq \epsilon .
\end{gathered}
$$

\section{Preliminary lemma for hypothesis (A3)}

Fix a number $d_{0} \in(0,1)$. There is a $C^{\infty}$-function $\phi_{0}: \mathbb{R}^{1} \rightarrow[0,1]$ such that $\phi_{0}(t)=1$ if $|t| \leq d_{0}, 1>\phi_{0}(t)>0$ if $d_{0}<|t|<1$, and $\phi_{0}(t)=0$ if $|t| \geq 1$. Define a $C^{\infty}$-function $\bar{\phi}$ : $\mathbb{R}^{1} \rightarrow \mathbb{R}^{1}$ by $\bar{\phi}(x)=\int_{0}^{x} \phi_{0}(t) d t, x \in \mathbb{R}^{1}$. Clearly $\bar{\phi}$ is monotone increasing, $\bar{\phi}(x)=x$ if $|x| \leq$ $d_{0}$ and

$$
\begin{gathered}
\bar{\phi}(x)=\bar{\phi}(1) \quad \text { if } x \geq 1, \quad \bar{\phi}(x)=\bar{\phi}(-1) \quad \text { if } x \leq-1 \\
d_{0}=\bar{\phi}\left(d_{0}\right) \leq \bar{\phi}(x) \leq \bar{\phi}(1) \leq 1 \quad \forall x \in\left(d_{0}, 1\right) .
\end{gathered}
$$


Now we define a set $\mathscr{L} \subset C_{l}\left(B_{1} \times B_{2}\right)$. In the case $m=1$ we set $\mathscr{L}=C_{l}\left(B_{1} \times B_{2}\right)$ and in the case $m>1$ denote by $\mathscr{L}$ a singleton $\{0\}$ where 0 is a function in $C_{l}\left(B_{1} \times B_{2}\right)$ which is identical zero. In the case $m>1$ for each $(f, \xi) \in M(A, U) \times \mathscr{L}$ and each $(x, u) \in X(A, U)$ we set

$$
I^{(f, \xi)}(x, u)=I^{(f)}(x, u)
$$

(see (4.15) and (4.16)). For each measurable set $E \subset \mathbb{R}^{m}$, each measurable set $E_{0} \subset E$, and each $h \in L^{1}(E)$, we set

$$
\|h\|_{L^{1}\left(E_{0}\right)}=\int_{E_{0}}|h(t)| d t
$$

Fix an integer $k \geq 1$. It is easy to verify that all partial derivatives of the functions $(x, y) \rightarrow$ $\bar{\phi}\left(|x-y|^{2}\right),(x, y) \in \mathbb{R}^{q} \times \mathbb{R}^{q}$ with $q=n, N$ up to the order $k$, are bounded (by some $\bar{d}>0)$.

For each $\gamma \in(0,1)$, choose $\epsilon_{0}(\gamma) \in(0, \gamma)$ such that

$$
E_{X}\left(8 \epsilon_{0}(\gamma)\right) \subset\left\{\left(\left(x_{1}, u_{1}\right),\left(x_{2}, u_{2}\right)\right) \in X(A, U) \times X(A, U): \rho\left(\left(x_{1}, u_{1}\right),\left(x_{2}, u_{2}\right)\right) \leq \gamma\right\}
$$

(see (4.8)) and

$$
\epsilon_{0}(\gamma)<4^{-1} \gamma(\bar{d}+2)^{-1}
$$

and choose

$$
\begin{gathered}
\epsilon_{1}(\gamma) \in\left(0, d_{0} \epsilon_{0}(\gamma)\right), \\
\delta(\gamma) \in\left(0,16^{-1} \epsilon_{1}(\gamma)^{4}\right) .
\end{gathered}
$$

The following result was established in [20].

Lemma 7.1. Let $\gamma \in(0,1), f \in M(A, U), \xi \in \mathscr{L}$, and let $Y \subset X(A, U),(\bar{x}, \bar{u}) \in Y$,

$$
I^{(f, \xi)}(\bar{x}, \bar{u}) \leq \inf \left\{I^{(f, \xi)}(x, u):(x, u) \in Y\right\}+\delta(\gamma)<\infty .
$$

Then there is $g: \mathbb{R}^{m} \times \mathbb{R}^{n} \times \mathbb{R}^{N} \rightarrow \mathbb{R}^{1}$ in $C^{k}\left(\mathbb{R}^{m+n+N}\right)$ which satisfies

$$
\begin{gathered}
0 \leq g(t, x, u) \leq \gamma \quad \forall(t, x, u) \in \mathbb{R}^{m} \times \mathbb{R}^{n} \times \mathbb{R}^{N}, \\
\|g(t, \cdot, \cdot)\|_{C^{k}\left(\mathbb{R}^{n} \times \mathbb{R}^{N}\right)} \leq \gamma \quad \forall t \in \mathbb{R}^{m}
\end{gathered}
$$

such that for a function $\bar{f} \in M(A, U)$ defined by

$$
\bar{f}(t, x, u)=f(t, x, u)+g(t, x, u), \quad(t, x, u) \in \operatorname{graph}(U),
$$

the following properties hold:

$$
I^{(\bar{f}, \bar{\xi})}(\bar{x}, \bar{u}) \leq I^{(f, \xi)}(\bar{x}, \bar{u})+\delta(\gamma) ;
$$


for each $(y, v) \in Y$ satisfying

$$
I^{(\bar{f}, \xi)}(y, v) \leq \inf \left\{I^{(\bar{f}, \xi)}(z, w):(z, w) \in Y\right\}+2 \delta(\gamma),
$$

the relation $\rho((y, v),(\bar{x}, \bar{u})) \leq \gamma$ is valid.

Moreover, the function $g$ is the sum of two functions, one of them depending only on $(t, x)$ while the other depending only on $(t, u)$.

\section{Auxiliary lemma for hypothesis (A4)}

Let $p \geq 1$ be an integer and let $e_{1}=(1,0, \ldots, 0), \ldots, e_{p}=(0, \ldots, 0,1)$ be the standard basis in $\mathbb{R}^{p}$. For each set $E \subset \mathbb{R}^{p}$, denote by $\operatorname{conv}(E)$ its convex hull. We have the following result (see [20, Proposition 6.1]).

Proposition 8.1. Let a finite set $E=\left\{h_{i j}: i=1,2, \ldots, p, j=1,2\right\} \subset \mathbb{R}^{p}$ satisfy

$$
\left|h_{i 1}-e_{i}\right|,\left|h_{i 2}+e_{i}\right| \leq(2 p)^{-1}, \quad i=1, \ldots, p .
$$

Then the relation $0 \in \operatorname{conv}(E)$ holds.

Assume that $A: \Omega \rightarrow 2^{\mathbb{R}^{n}} \backslash\{\varnothing\}$ and $\operatorname{graph}(A)$ is a closed subset of the space $\Omega \times \mathbb{R}^{n}$ with the product topology. Let $e_{1}=(1,0, \ldots, 0), e_{2}=(0,1, \ldots, 0), \ldots, e_{N}=(0,0, \ldots, 1)$ be a standard basis in $\mathbb{R}^{N}$. Now we define a set $\mathscr{L} \subset C_{l}\left(B_{1} \times B_{2}\right)$. In the case $m=1$ we set $\mathscr{L}=$ $C_{l}\left(B_{1} \times B_{2}\right)$ and in the case $m>1$ we denote by $\mathscr{L}$ a singleton $\{0\}$ where 0 is a function in $C_{l}\left(B_{1} \times B_{2}\right)$ which is identical zero. In the case $m>1$ for each $(f, \xi) \in M\left(A, \tilde{U}_{A}\right) \times \mathscr{L}$ and each $(x, u) \in X\left(A, \tilde{U}_{A}\right)$ we set

$$
I^{(f, \xi)}(x, u)=I^{(f)}(x, u)
$$

(see (4.15), (4.16), and (4.17)).

The following result was established in [20].

Lemma 8.2. Let $f \in M\left(A, \tilde{U}_{A}\right), \xi \in \mathscr{L}, U \in \mathscr{P}_{A}$,

$$
\left\{(x, u) \in X(A, U): I^{(f, \xi)}(x, u)<\infty\right\} \neq \varnothing,
$$

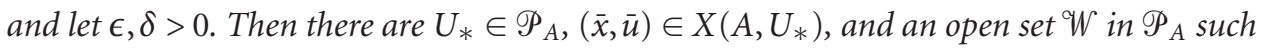
that

$$
\begin{gathered}
\left(U_{*}, U\right) \in E_{\mathscr{P}_{A}}(\epsilon), \quad \mathscr{W} \cap\left\{V \in \mathscr{P}_{A}:(U, V) \in E_{\mathscr{P}_{A}}(\epsilon)\right\} \neq \varnothing, \\
I^{(f, \xi)}(\bar{x}, \bar{u}) \leq \inf \left\{I^{(f, \xi)}(x, u):(x, u) \in X\left(A, U_{*}\right)\right\}+\delta<\infty
\end{gathered}
$$

and for all $V \in \mathcal{W}$,

$$
(\bar{x}, \bar{u}) \in X(A, V) \subset X\left(A, U_{*}\right)
$$

\section{Proof of Theorem 4.2 and its extensions}

Proof of Theorem 4.2. By Propositions 6.1 and 6.2, (A1) holds and $J_{a}$ is lower semicontinuous for all $a \in \mathscr{A}_{1} \times \mathscr{A}_{2}$. By Theorem 5.1, we need to verify that (H1) and (H2) are 
valid. Hypothesis (H2) follows from Proposition 6.2. Therefore it is sufficient to show that (H1) holds. By Proposition 5.3, it is sufficient to show that (A2), (A3), and (A4) are valid. Hypothesis (A2) follows from Propositions 6.5 and 6.7. By Lemma 7.1, (A3) holds. Hypothesis (A4) follows from Lemma 8.2. This completes the proof of the theorem.

Now we present the extension of Theorem 4.2.

Assume that $A: \Omega \rightarrow 2^{\mathbb{R}^{n}} \backslash\{\varnothing\}, U: \operatorname{graph}(A) \rightarrow 2^{\mathbb{R}^{N}} \backslash\{\varnothing\}$ and $\operatorname{graph}(U)$ is a closed subset of $\Omega \times \mathbb{R}^{n} \times \mathbb{R}^{N}$ with the product topology. We consider the metric space $X(A, U)$ with the metric $\rho$ (see (4.8)).

Now we define $\mathscr{A}_{1}$ as follows:

$$
\mathscr{A}_{1}=\mathscr{A}_{11} \times \mathscr{A}_{12} \quad \text { if } m=1, \quad \mathscr{A}_{1}=\mathscr{A}_{11} \quad \text { if } m>1,
$$

where $\mathscr{A}_{12}$ is either $C_{l}\left(B_{1} \times B_{2}\right)$ or $C\left(B_{1} \times B_{2}\right)$ or a singleton $\{\xi\} \subset C_{l}\left(B_{1} \times B_{2}\right)$, and $\mathscr{A}_{11}$ is one of the following spaces:

$$
\begin{array}{ccc}
M(A, U), & M^{l}(A, U), & M^{c}(A, U), \\
M_{k}\left(A, \tilde{U}_{A}\right), & M_{k}^{l}\left(A, \tilde{U}_{A}\right), & M_{k}^{c}\left(A, \tilde{U}_{A}\right)
\end{array}
$$

(here $k \geq 1$ is an integer, $U=\widetilde{U}_{A}$, and $\operatorname{graph}(A)$ is a closed subset of the space $\Omega \times \mathbb{R}^{n}$ with the product topology),

$$
M_{k}^{*}(\tilde{A}, \tilde{U}), \quad M_{k}^{* l}(\tilde{A}, \tilde{U}), \quad M_{k}^{* c}(\tilde{A}, \tilde{U})
$$

(here $k \geq 1$ is an integer and $A=\tilde{A}, U=\tilde{U}$ ).

Denote by $\mathscr{A}$ the closure of the set $\left\{a \in \mathscr{A}_{1}: \inf \left(I^{(a)}\right)<\infty\right\}$ in the space $\mathscr{A}_{1}$ with the strong topology. We assume that $\mathscr{A}$ is nonempty. The following result is proved analogously to Theorem 4.2 .

THeorem 9.1. There exists an everywhere dense (in the strong topology) set $\mathscr{B} \subset A$ which is

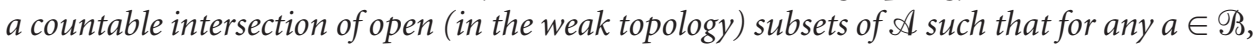
the following assertions hold:

(1) $\inf \left(I^{(a)}\right)$ is finite and attained at a unique pair $(\bar{x}, \bar{u}) \in X(A, U)$,

(2) for each $\epsilon>0$, there are a neighborhood $\mathscr{V}$ of a in $\mathcal{A}$ with the weak topology and $\delta>0$ such that for each $b \in \mathscr{V}, \inf \left(I^{(b)}\right)$ is finite and if $(z, w) \in X(A, U)$ satisfies $I^{(b)}(z, w) \leq \inf \left(I^{(b)}\right)+\delta$, then $\rho((\bar{x}, \bar{u}),(z, w)) \leq \epsilon$ and $\left|I^{(b)}(z, w)-I^{(a)}(\bar{x}, \bar{u})\right| \leq \epsilon$.

In the sequel, we use the notation and definitions from Sections 4 and 5. Let $m, n, N \geq 1$ be integers. We again assume that $\Omega$ is a fixed bounded domain in $\mathbb{R}^{m}, H(t, x, u)$ is a fixed continuous function defined on $\Omega \times \mathbb{R}^{n} \times \mathbb{R}^{N}$ with values in $\mathbb{R}^{m n}$ such that $H(t, x, u)=$ $\left(H_{i}\right)_{i=1}^{n}$ and $H_{i}=\left(H_{i j}\right)_{j=1}^{m}, i=1, \ldots, n, B_{1}$ and $B_{2}$ are fixed nonempty closed subsets of $\mathbb{R}^{n}$ and $\theta^{*}=\left(\theta_{i}^{*}\right)_{i=1}^{n} \in\left(W^{1,1}(\Omega)\right)^{n}$ is also fixed. If $m=1$, then we assume that $\Omega=\left(T_{1}, T_{2}\right)$, where $T_{1}$ and $T_{2}$ are fixed real numbers for which $T_{1}<T_{2}$.

Define set-valued mappings $\tilde{A}: \Omega \rightarrow 2^{\mathbb{R}^{n}} \backslash\{\varnothing\}$ and $\tilde{U}: \Omega \times \mathbb{R}^{n} \rightarrow 2^{\mathbb{R}^{N}} \backslash\{\varnothing\}$ by

$$
\widetilde{A}(t)=\mathbb{R}^{n}, \quad t \in \Omega, \quad \tilde{U}(t, x)=\mathbb{R}^{N}, \quad(t, x) \in \Omega \times \mathbb{R}^{n} .
$$


Consider the metric space $(X(\tilde{A}, \tilde{U}), \rho)$ (see $(4.7))$ and the spaces of integrands

$$
\begin{aligned}
& \mu(\tilde{A}, \tilde{U}), \quad \mu^{l}(\tilde{A}, \tilde{U}), \quad \mu^{c}(\tilde{A}, \tilde{U}), \\
& M_{k}(\tilde{A}, \tilde{U}), \quad M_{k}^{l}(\tilde{A}, \tilde{U}), \quad M_{k}^{c}(\tilde{A}, \tilde{U}), \\
& M_{k}^{*}(\tilde{A}, \tilde{U}), \quad M_{k}^{* l}(\tilde{A}, \tilde{U}), \quad M_{k}^{* c}(\tilde{A}, \tilde{U}) \quad \text { (here } k \geq 1 \text { is an integer) }
\end{aligned}
$$

defined in Section 4.

Denote by $S\left(\mathbb{R}^{n} \times \mathbb{R}^{N}\right)$ the set of all nonempty convex closed subsets of $\mathbb{R}^{n} \times \mathbb{R}^{N}=$ $\mathbb{R}^{n+N}$. For each $x \in \mathbb{R}^{n} \times \mathbb{R}^{N}$ and each $E \subset \mathbb{R}^{n} \times \mathbb{R}^{N}$, set $d_{H}(x, E)=\inf _{y \in E}|x-y|$. For each pair of sets $C_{1}, C_{2} \subset \mathbb{R}^{n} \times \mathbb{R}^{N}$,

$$
d_{H}\left(C_{1}, C_{2}\right)=\max \left\{\sup _{y \in C_{1}} d_{H}\left(y, C_{2}\right), \sup _{x \in C_{2}} d_{H}\left(x, C_{1}\right)\right\}
$$

is the Hausdorff distance between $C_{1}$ and $C_{2}$. For the space $S\left(\mathbb{R}^{n} \times \mathbb{R}^{N}\right)$, we consider the uniformity determined by the following base:

$$
E_{\mathbb{R}^{n+N}}(\epsilon)=\left\{\left(C_{1}, C_{2}\right) \in S\left(\mathbb{R}^{n} \times \mathbb{R}^{N}\right) \times S\left(\mathbb{R}^{n} \times \mathbb{R}^{N}\right): d_{H}\left(C_{1}, C_{2}\right) \leq \epsilon\right\},
$$

where $\epsilon>0$. It is well known that the space $S\left(\mathbb{R}^{n} \times \mathbb{R}^{N}\right)$ with this uniformity is metrizable and complete (see Section 4 ). Denote by $\mathscr{P}$ the set of all set-valued mappings $Q: \Omega \rightarrow$ $S\left(\mathbb{R}^{n} \times \mathbb{R}^{N}\right)$ such that the $\operatorname{graph}(Q)$ is a closed subset of the space $\Omega \times \mathbb{R}^{n} \times \mathbb{R}^{N}$ with the product topology. For each $Q \in \mathscr{P}$, define $A_{Q}: \Omega \rightarrow 2^{\mathbb{R}^{n}} \backslash\{\varnothing\}$ by

$$
A_{Q}(t)=\left\{x \in \mathbb{R}^{n}: \text { there is } u \in \mathbb{R}^{N} \text { such that }(t, x, u) \in \operatorname{graph}(Q)\right\}
$$

and a set-valued mapping $U_{Q}: \operatorname{graph}\left(A_{Q}\right) \rightarrow 2^{\mathbb{R}^{n}} \backslash\{\varnothing\}$ by

$$
U_{Q}(t, x)=\left\{u \in \mathbb{R}^{N}:(t, x, u) \in \operatorname{graph}(Q)\right\}, \quad(t, x) \in \operatorname{graph}\left(A_{Q}\right) .
$$

For the space $\mathscr{P}$, we consider the uniformity determined by the following base:

$$
E_{\mathscr{P}}(\epsilon)=\left\{\left(Q_{1}, Q_{2}\right) \in \mathscr{P} \times \mathscr{P}: d_{H}\left(Q_{1}(t), Q_{2}(t)\right) \leq \epsilon, t \in \Omega\right\},
$$

where $\epsilon>0$. It is not difficult to verify that the space $\mathscr{P}$ with this uniformity is metrizable and complete. We equip the set $\mathscr{P}$ with the topology induced by this uniformity.

For each $Q \in \mathscr{P}$, define

$$
S_{Q}=X\left(A_{Q}, U_{Q}\right)=\{(x, u) \in X(\tilde{A}, \tilde{U}):(x(t), u(t)) \in Q(t), t \in \Omega \text { (a.e.) }\} .
$$

In the case $m=1$ for each $Q \in \mathscr{P}$ and each $(f, \xi) \in \mathcal{M}(\tilde{A}, \tilde{U}) \times C_{l}\left(B_{1} \times B_{2}\right)$ we consider the optimal control problem

$$
I^{(f, \xi)}(x, u) \longrightarrow \min , \quad(x, u) \in X\left(A_{Q}, U_{Q}\right)
$$

and in the case $m>1$ for each $Q \in \mathscr{P}$ and each $f \in \mathcal{M}(\tilde{A}, \tilde{U})$ we consider the optimal control problem

$$
I^{(f)}(x, u) \longrightarrow \min , \quad(x, u) \in X\left(A_{Q}, U_{Q}\right)
$$


We set $\mathscr{A}_{2}=\mathscr{P}$ and define a space $\mathscr{A}_{1}$ as follows:

$$
\mathscr{A}_{1}=\mathscr{A}_{11} \times \mathscr{A}_{12} \quad \text { if } m=1, \quad \mathscr{A}_{1}=\mathscr{A}_{11} \quad \text { if } m>1
$$

where $\mathscr{A}_{12}$ is either $C_{l}\left(B_{1} \times B_{2}\right)$ or $C\left(B_{1} \times B_{2}\right)$ or a singleton $\{\xi\} \subset C_{l}\left(B_{1} \times B_{2}\right)$, and $\mathscr{A}_{11}$ is one of the following spaces:

$$
\begin{aligned}
& \mu(\tilde{A}, \tilde{U}), \quad \mu^{l}(\tilde{A}, \tilde{U}), \quad \mu^{c}(\tilde{A}, \tilde{U}), \\
& \mu_{k}(\tilde{A}, \tilde{U}), \quad \mu_{k}^{l}(\tilde{A}, \tilde{U}), \quad \mu_{k}^{c}(\tilde{A}, \tilde{U}), \\
& M_{k}^{*}(\tilde{A}, \tilde{U}), \quad M_{k}^{* l}(\tilde{A}, \tilde{U}), \quad M_{k}^{* c}(\tilde{A}, \tilde{U}) \quad \text { (here } k \geq 1 \text { is an integer). }
\end{aligned}
$$

For each $a=\left(a_{1}, a_{2}\right) \in \mathscr{A}_{1} \times \mathscr{A}_{2}$, we define $J_{a}: X(\tilde{A}, \tilde{U}) \rightarrow \mathbb{R}^{1} \cup\{\infty\}$ by

$$
J_{a}(x, u)=I^{\left(a_{1}\right)}(x, u), \quad(x, u) \in S_{a_{2}}, \quad J_{a}(x, u)=\infty, \quad(x, u) \in X(\tilde{A}, \tilde{U}) \backslash S_{a_{2}} .
$$

By Propositions 6.1 and 6.2, $J_{a}$ is lower semicontinuous for all $a \in \mathscr{A}_{1} \times A_{2}$. Denote by $\mathscr{A}$ the closure of the set $\left\{a \in \mathscr{A}_{1} \times \mathscr{A}_{2}: \inf \left(J_{a}\right)<\infty\right\}$ in the space $\mathscr{A}_{1} \times A_{2}$ with the strong topology. We assume that $\mathscr{A}$ is nonempty. The following result is an extension of Theorem 4.2.

THEOREM 9.2. There exists an everywhere dense (in the strong topology) set $\mathscr{B} \subset \mathscr{A}$ which is a countable intersection of open (in the weak topology) subsets of $\mathscr{A}$ such that for any $a \in \mathscr{B}$, the following assertions hold:

(1) $\inf \left(J_{a}\right)$ is finite and attained at a unique pair $(\bar{x}, \bar{u}) \in X(\tilde{A}, \tilde{U})$,

(2) for each $\epsilon>0$, there are a neighborhood $\mathscr{V}$ of $a$ in $\mathcal{A}$ with the weak topology and $\delta>0$ such that for each $b \in \mathscr{V}, \inf \left(J_{b}\right)$ is finite and if $(z, w) \in X(\tilde{A}, \tilde{U})$ satisfies $J_{b}(z, w) \leq$ $\inf \left(J_{b}\right)+\delta$, then $\rho((\bar{x}, \bar{u}),(z, w)) \leq \epsilon$ and $\left|J_{b}(z, w)-J_{a}(\bar{x}, \bar{u})\right| \leq \epsilon$.

Proof of Theorem 9.2. By Propositions 6.1 and 6.2 (A1) holds and $J_{a}$ is lower semicontinuous for all $a \in \mathscr{A}_{1} \times \mathscr{A}_{2}$. By Theorem 5.1 we need to verify that (H1) and (H2) are valid. Hypothesis (H2) follows from Proposition 6.2. Therefore it is sufficient to show that $(\mathrm{H} 1)$ holds. By Proposition 5.3 it is sufficient to show that (A2), (A3), and (A4) are valid. Hypothesis (A2) follows from Propositions 6.5 and 6.7. By Lemma 7.1 (A3) holds. It is easy to see that (A4) follows from Lemma 9.3 proved below. Its proof is a modification of the proof of Lemma 8.2.

Let $e_{1}=(1,0, \ldots, 0), e_{2}=(0,1,0, \ldots, 0), \ldots, e_{n+N}=(0, \ldots, 0,1)$ be a standard basis in $\mathbb{R}^{n+N}$. As in Section 8 we define a set $\mathscr{L} \subset C_{l}\left(B_{1} \times B_{2}\right)$. In the case $m=1$ we set $\mathscr{L}=$ $C_{l}\left(B_{1} \times B_{2}\right)$ and in the case $m>1$ we denote by $\mathscr{L}$ a singleton $\{0\}$ where 0 is a function in $C_{l}\left(B_{1} \times B_{2}\right)$ which is identical zero. In the case $m>1$ for each $(f, \xi) \in M(\tilde{A}, \tilde{U}) \times \mathscr{L}$ and each $(x, u) \in X(\tilde{A}, \tilde{U})$ we set

$$
I^{(f, \xi)}(x, u)=I^{f}(x, u)
$$

(see (4.15), (4.16), and (4.17)). 
396 Solutions of optimal control problems

Lemma 9.3. Let $f \in M(\tilde{A}, \tilde{U}), \xi \in \mathscr{L}, Q \in \mathscr{P}$,

$$
\left\{(x, u) \in X\left(A_{Q}, U_{Q}\right): I^{(f, \xi)}(x, u)<\infty\right\} \neq \varnothing,
$$

$\epsilon, \delta>0$. Then there are $Q_{*} \in \mathscr{P},(\bar{x}, \bar{u}) \in X\left(A_{Q_{*}}, U_{Q_{*}}\right)$, an open set $\mathscr{W}$ in $\mathscr{P}$ such that

$$
\begin{gathered}
\left(Q, Q_{*}\right) \in E_{\mathscr{P}}(\epsilon), \quad \mathscr{W} \cap\left\{P \in \mathscr{P}:(P, Q) \in E_{\mathscr{P}}(\epsilon)\right\} \neq \varnothing, \\
I^{(f, \xi)}(\bar{x}, \bar{u}) \leq \inf \left\{I^{(f, \xi)}(x, u):(x, u) \in X\left(A_{Q_{*}}, U_{Q_{*}}\right)\right\}+\delta<\infty
\end{gathered}
$$

and for each $P \in \mathcal{W}$,

$$
(\bar{x}, \bar{u}) \in X\left(A_{p}, U_{p}\right) \subset X\left(A_{Q_{*}}, U_{Q_{*}}\right) .
$$

Proof. For each $r \in(0,1]$, define $Q_{r} \in \mathscr{P}$ by

$$
Q_{r}(t)=\left\{(x, u) \in \mathbb{R}^{n} \times \mathbb{R}^{N}: d_{H}((x, u), Q(t)) \leq r\right\}, \quad t \in \Omega
$$

and define

$$
\mu(r)=\inf \left\{I^{(f, \xi)}(x, u):(x, u) \in X\left(A_{Q_{r}}, U_{Q_{r}}\right)\right\} .
$$

Clearly $\mu(r)$ is finite for all $r \in(0,1]$ and the function $\mu$ is monotone decreasing. There is $r_{0} \in\left(0,8^{-1} \epsilon\right)$ such that $\mu$ is continuous at $r_{0}$. Choose $r_{1} \in\left(0, r_{0}\right)$ such that

$$
\left|\mu\left(r_{1}\right)-\mu\left(r_{0}\right)\right|<16^{-1} \delta .
$$

There is $(\bar{x}, \bar{u}) \in X\left(A_{Q_{r_{1}}}, U_{Q_{r_{1}}}\right)$ such that

$$
I^{(f, \xi)}(\bar{x}, \bar{u}) \leq \mu\left(r_{1}\right)+16^{-1} \delta .
$$

Relations (9.22), (9.23), and (9.24) imply that

$$
I^{(f, \xi)}(\bar{x}, \bar{u}) \leq \mu\left(r_{0}\right)+8^{-1} \delta .
$$

Set

$$
r_{2}=\frac{r_{0}+r_{1}}{2}
$$

Clearly

$$
\left(Q_{r_{i}}, Q\right) \in E_{\mathscr{P}}(\epsilon), \quad i=0,1,2 .
$$

Choose a positive number

$$
\gamma<\min \left\{4^{-1} \delta,(16(n+N))^{-1}\left(r_{0}-r_{1}\right)\right\}
$$

and define

$$
\mathscr{W}=\left\{P \in \mathscr{P}:\left(Q_{r_{2}}, P\right) \in E_{\mathscr{P}}(\gamma)\right\} .
$$


Assume that $P \in \mathcal{W}$. By (9.29), (9.21), (9.28), and (9.26) for all $t \in \Omega$,

$$
\begin{aligned}
P(t) & \subset\left\{(x, u) \in \mathbb{R}^{n} \times \mathbb{R}^{N}: d_{H}\left((x, u), Q_{r_{2}}(t)\right) \leq \gamma\right\} \\
& \subset\left\{(x, u) \in \mathbb{R}^{n} \times \mathbb{R}^{N}: d_{H}((x, u), Q(t)) \leq r_{0}\right\} \subset Q_{r_{0}}(t) .
\end{aligned}
$$

Therefore $X\left(A_{p}, U_{p}\right) \subset X\left(A_{Q_{r_{0}}}, U_{Q_{r_{0}}}\right)$. We will show that $(\bar{x}, \bar{u}) \in X\left(A_{p}, U_{p}\right)$. It is sufficient to show that for a.e. $t \in \Omega,(t, \bar{x}(t), \bar{u}(t)) \in \operatorname{graph}(P)$.

Since $(\bar{x}, \bar{u}) \in X\left(A_{Q_{r_{1}}}, U_{Q_{r_{1}}}\right)$ for almost all $t \in \Omega$,

$$
(t, \bar{x}(t), \bar{u}(t)) \in Q_{r_{1}}(t)
$$

Assume that $t \in \Omega$ and (9.31) is valid. It follows from (9.31), (9.26), and (9.29) that for $i=1, \ldots, n+N$,

$$
(\bar{x}(t), \bar{u}(t))+2^{-1}\left(r_{0}-r_{1}\right) e_{i},(\bar{x}(t), \bar{u}(t))-2^{-1}\left(r_{0}-r_{1}\right) e_{i} \in Q_{r_{2}}(t),
$$

and there are $z_{i 1}, z_{i 2} \in \mathbb{R}^{n+N}$ such that

$$
\begin{gathered}
(\bar{x}(t), \bar{u}(t))+z_{i 1},(\bar{x}(t), \bar{u}(t))+z_{i 2} \in P(t), \\
\left|z_{i 1}-2^{-1}\left(r_{0}-r_{1}\right) e_{i}\right|,\left|z_{i 2}+2^{-1}\left(r_{0}-r_{1}\right) e_{i}\right| \leq \gamma .
\end{gathered}
$$

Since $P(t)$ is convex, it follows from these relations, (9.28) and Proposition 8.1 that $0 \in$ $\operatorname{conv}\left\{z_{i 1}, z_{i 2}: i=1, \ldots, n+N\right\}$ and therefore $(\bar{x}(t), \bar{u}(t)) \in P(t)$. Thus $(\bar{x}, \bar{u}) \in X\left(A_{p}, U_{p}\right)$. This completes the proof of the lemma and the proof of Theorem 9.2.

\section{An extension of Theorem 4.2}

In this section, we use the notation and definitions from Sections 4 and 5 .

Let $m=1$ and let $n, N \geq 1$ be integers, $B_{1}, B_{2}=\mathbb{R}^{n}, \Omega=\left(T_{1}, T_{2}\right)$, where $T_{1}$ and $T_{2}$ are fixed real numbers for which $T_{1}<T_{2}$, and let $H(t, x, u)$ be a fixed continuous function defined on $\Omega \times \mathbb{R}^{n} \times \mathbb{R}^{N}$ with values in $\mathbb{R}^{n}$ such that $H(t, x, u)=\left(H_{i}\right)_{i=1}^{n}$.

Consider a fixed set-valued mapping $A: \Omega \rightarrow 2^{\mathbb{R}^{n}} \backslash\{\varnothing\}$ for which $\operatorname{graph}(A)$ is a closed subset of the space $\Omega \times \mathbb{R}^{n}$ with the product topology and a set-valued mapping $\tilde{U}_{A}$ : $\operatorname{graph}(A) \rightarrow 2^{\mathbb{R}^{N}}$ defined by

$$
\tilde{U}_{A}(t, x)=\mathbb{R}^{N}, \quad(t, x) \in \operatorname{graph}(A)
$$

(see (4.17)). We consider the metric space $X\left(A, \tilde{U}_{A}\right)$ with the metric $\rho$ (see (4.8)), the uniform space $\mathscr{P}_{A}$, and the space of integrands $\mathcal{M}\left(A, \widetilde{U}_{A}\right)$ and all its subspaces introduced in Section 4 . Note that all of these spaces are equipped with the corresponding uniformities and topologies introduced in Section 4.

Denote by $S\left(\mathbb{R}^{n}\right)$ the set of all nonempty convex closed subsets of $\mathbb{R}^{n}$. For each $x \in \mathbb{R}^{n}$ and each $E \subset \mathbb{R}^{n}$, set $d_{H}(x, E)=\inf _{y \in E}|x-y|$. For each pair of sets $C_{1}, C_{2} \subset \mathbb{R}^{n}$,

$$
d_{H}\left(C_{1}, C_{2}\right)=\max \left\{\sup _{y \in C_{1}} d_{H}\left(y, C_{2}\right), \sup _{x \in C_{2}} d_{H}\left(x, C_{1}\right)\right\}
$$


is the Hausdorff distance between $C_{1}$ and $C_{2}$. For the space $S\left(\mathbb{R}^{n}\right)$, we consider the uniformity determined by the following base:

$$
E_{\mathbb{R}^{n}}(\epsilon)=\left\{\left(C_{1}, C_{2}\right) \in S\left(\mathbb{R}^{n}\right) \times S\left(\mathbb{R}^{n}\right): d_{H}\left(C_{1}, C_{2}\right) \leq \epsilon\right\},
$$

where $\epsilon>0$. It is well known that the space $S\left(\mathbb{R}^{n}\right)$ with this uniformity is metrizable and complete.

For each $\left(C_{1}, C_{2}, U\right) \in S\left(\mathbb{R}^{n}\right) \times S\left(\mathbb{R}^{n}\right) \times \mathscr{P}_{A}$, define

$$
S_{C_{1} C_{2} U}=\left\{(x, u) \in X\left(A, \tilde{U}_{A}\right): u(t) \in U(t, x(t)), t \in \Omega \text { a.e. and } x\left(T_{i}\right) \in C_{i}, i=1,2\right\} .
$$

For each $C_{1}, C_{2} \in S\left(\mathbb{R}^{n}\right)$, each $U \in \mathscr{P}_{A}$, and each $(f, \xi) \in M(A, \tilde{U}) \times C_{l}\left(\mathbb{R}^{n} \times \mathbb{R}^{n}\right)$, we consider the optimal control problem

$$
I^{(f, \xi)}(x, u) \longrightarrow \min , \quad(x, u) \in S_{C_{1}, C_{2} U} .
$$

We set $\mathscr{A}_{2}=S\left(\mathbb{R}^{n}\right) \times S\left(\mathbb{R}^{n}\right) \times \mathscr{P}_{A}$ and define a space $\mathscr{A}_{1}$ as follows.

$\mathscr{A}_{1}=\mathscr{A}_{11} \times \mathscr{A}_{12}$ where $\mathscr{A}_{12}$ is either $C_{l}\left(\mathbb{R}^{n} \times \mathbb{R}^{n}\right)$ or $C\left(\mathbb{R}^{n} \times \mathbb{R}^{n}\right)$ or a singleton $\{\xi\} \subset$ $C_{l}\left(\mathbb{R}^{N} \times \mathbb{R}^{N}\right)$, and $\mathscr{A}_{11}$ is one of the following spaces:

$$
\begin{gathered}
M\left(A, \tilde{U}_{A}\right) ; \quad M^{l}\left(A, \tilde{U}_{A}\right) ; \quad M^{c}\left(A, \tilde{U}_{A}\right) ; \\
\mathcal{M}_{k}\left(A, \tilde{U}_{A}\right) ; \quad M_{k}^{l}\left(A, \tilde{U}_{A}\right) ; \quad M_{k}^{c}\left(A, \tilde{U}_{A}\right) \quad \text { (here } k \geq 1 \text { is an integer); } \\
\left.M_{k}^{*}(\tilde{A}, \tilde{U}) ; \quad M_{k}^{* l}(\tilde{A}, \tilde{U}) ; \quad M_{k}^{* c}(\tilde{A}, \tilde{U}) \quad \text { (here } k \geq 1 \text { is an integer and } A=\tilde{A}\right) .
\end{gathered}
$$

For each $a=\left(a_{1}, a_{2}\right) \in \mathscr{A}_{1} \times \mathscr{A}_{2}$, we define $J_{a}: X\left(A, \tilde{U}_{A}\right) \rightarrow \mathbb{R}^{1} \cup\{\infty\}$ by

$$
\begin{gathered}
J_{a}(x, u)=I^{\left(a_{1}\right)}(x, u), \quad(x, u) \in S_{a_{2}}, \\
J_{a}(x, u)=\infty, \quad(x, u) \in X\left(A, \widetilde{U}_{A}\right) \backslash S_{a_{2}} .
\end{gathered}
$$

By Propositions 6.1 and $6.2 J_{a}$ is lower semicontinuous for all $a \in \mathscr{A}_{1} \times \mathscr{A}_{2}$. Denote by $\mathscr{A}$ the closure of the set $\left\{a \in \mathscr{A}_{1} \times \mathscr{A}_{2}: \inf \left(J_{a}\right)<\infty\right\}$ in the space $\mathscr{A}_{1} \times \mathscr{A}_{2}$ with the strong topology. We assume that $\mathscr{A}$ is nonempty. We prove the following result.

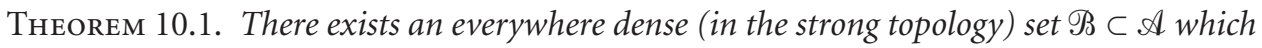
is a countable intersection of open (in the weak topology) subsets of $\mathscr{A}$ such that for any $a \in \mathscr{B}$, the following assertions hold:

(1) $\inf \left(J_{a}\right)$ is finite and attained at a unique pair $(\bar{x}, \bar{u}) \in X\left(A, \tilde{U}_{A}\right)$,

(2) for each $\epsilon>0$, there are a neighborhood $\mathscr{V}$ of $a$ in $\mathscr{A}$ with the weak topology and $\delta>0$ such that for each $b \in \mathscr{V}, \inf \left(J_{b}\right)$ is finite and if $(z, w) \in X\left(A, \tilde{U}_{A}\right)$ satisfies $J_{b}(z, w) \leq \inf \left(J_{b}\right)+\delta$, then $\rho((\bar{x}, \bar{u}),(z, w)) \leq \epsilon$ and $\left|J_{b}(z, w)-J_{a}(\bar{x}, \bar{u})\right| \leq \epsilon$.

Proof of Theorem 10.1. By Propositions 6.1 and 6.2 (A1) holds and $J_{a}$ is lower semicontinuous for all $a \in \mathscr{A}_{1} \times \mathscr{A}_{2}$. By Theorem 5.1 we need to verify that (H1) and (H2) are valid. Hypothesis (H2) follows from Proposition 6.2. Therefore it is sufficient to show that (H1) holds. By Proposition 5.3 it is sufficient to show that (A2), (A3), and (A4) are 
valid. Hypothesis (A2) follows from Proposition 6.7. By Lemma 7.1 (A3) holds. It is easy to see that (A4) follows from Lemma 10.2 proved below. Its proof is a modification of the proof of Lemma 8.2.

Lemma 10.2. Let $f \in M\left(A, \tilde{U}_{A}\right), \xi \in C_{l}\left(\mathbb{R}^{n} \times \mathbb{R}^{n}\right), U \in \mathscr{P}_{A}, C_{1}, C_{2} \in S\left(\mathbb{R}^{n}\right)$,

$$
\left\{(x, u) \in S_{C_{1} C_{2} U}: I^{(f, \xi)}(x, u)<\infty\right\} \neq \varnothing,
$$

and let $\epsilon, \delta>0$. Then there are $U_{*} \in \mathscr{P}_{A}, C_{* 1}, C_{* 2} \in S\left(\mathbb{R}^{n}\right)$,

$$
(\bar{x}, \bar{u}) \in S_{C_{* 1} C_{* 2} U_{*}},
$$

and an open set $\mathscr{W}$ in $S\left(\mathbb{R}^{n}\right) \times S\left(\mathbb{R}^{n}\right) \times \mathscr{P}_{A}$ such that

$$
\begin{gathered}
\left(U_{*}, U\right) \in E_{\mathscr{P}_{A}}(\epsilon), \quad\left(C_{i}, C_{* i}\right) \in E_{\mathbb{R}^{n}}(\epsilon), \quad i=1,2, \\
W \cap\left\{\left(D_{1}, D_{2}, V\right):\left(D_{i}, C_{i}\right) \in E_{\mathbb{R}^{n}}(\epsilon), i=1,2,(V, U) \in E_{\mathscr{P}_{A}}(\epsilon)\right\} \neq \varnothing, \\
I^{(f, \xi)}(\bar{x}, \bar{u}) \leq \inf \left\{I^{(f, \xi)}(x, u):(x, u) \in S_{C_{* 1}, C_{* 2} U_{*}}\right\}+\delta<\infty
\end{gathered}
$$

and for all $\left(D_{1}, D_{2}, V\right) \in \mathcal{W}$,

$$
(\bar{x}, \bar{u}) \in S_{D_{1} D_{2} V} \subset S_{C_{* 1} C_{* 2} U_{*}} .
$$

Proof. For each $r \in(0,1]$, define $U_{r} \in \mathscr{P}_{A}$ by

$$
\begin{gathered}
U_{r}(t, x)=\left\{u \in \mathbb{R}^{N}: d_{H}(u, U(t, x)) \leq r\right\}, \quad(t, x) \in \operatorname{graph}(A), \\
C_{r 1}=\left\{z \in \mathbb{R}^{n}: d_{H}\left(z, C_{1}\right) \leq r\right\}, \\
C_{r 2}=\left\{z \in \mathbb{R}^{n}: d_{H}\left(z, C_{2}\right) \leq r\right\}
\end{gathered}
$$

and define

$$
\mu(r)=\inf \left\{I^{(f, \xi)}(x, u):(x, u) \in S_{C_{r 1} C_{r 2} U_{r}}\right\} .
$$

Clearly $\mu(r)$ is finite for all $r \in(0,1]$ and the function $\mu$ is monotone decreasing. There is $r_{0} \in\left(0,8^{-1} \epsilon\right)$ such that $\mu$ is continuous at $r_{0}$. Choose $r_{1} \in\left(0, r_{0}\right)$ such that

$$
\left|\mu\left(r_{1}\right)-\mu\left(r_{0}\right)\right|<16^{-1} \delta .
$$

There is

$$
(\bar{x}, \bar{u}) \in S_{C_{r_{1} 1} C_{r_{1} 2} U_{r_{1}}}
$$

such that

$$
I^{(f, \xi)}(\bar{x}, \bar{u}) \leq \mu\left(r_{1}\right)+16^{-1} \delta .
$$

Relations (10.14), (10.17), and (10.15) imply that

$$
I^{(f, \xi)}(\bar{x}, \bar{u}) \leq \mu\left(r_{0}\right)+8^{-1} \delta .
$$


Set

$$
r_{2}=\frac{r_{0}+r_{1}}{2}
$$

Clearly

$$
\begin{gathered}
\left(U_{r_{i}}, U\right) \in E_{\mathscr{P}_{A}}(\epsilon), \quad i=0,1,2, \\
\left(C_{r_{i} 1}, C_{1}\right),\left(C_{r_{i} 2}, C_{2}\right) \in E_{\mathbb{R}^{n}}(\epsilon), \quad i=0,1,2 .
\end{gathered}
$$

Choose a positive number

$$
\gamma<\min \left\{4^{-1} \delta,(16(n+N))^{-1}\left(r_{0}-r_{1}\right)\right\}
$$

and define

$$
\begin{aligned}
\mathscr{W}=\{ & \left(D_{1}, D_{2}, P\right) \in S\left(\mathbb{R}^{n}\right) \times S\left(\mathbb{R}^{n}\right) \times \mathscr{P}_{A}: \\
& \left.\left(U_{r_{2}}, P\right) \in E_{\mathscr{P}_{A}}(\gamma),\left(D_{1}, C_{r_{2} 1}\right),\left(D_{2}, C_{r_{2} 2}\right) \in E_{\mathbb{R}^{n}}(\gamma)\right\} .
\end{aligned}
$$

Assume that

$$
\left(D_{1}, D_{2}, V\right) \in \mathcal{W} \text {. }
$$

By (10.23), (10.22), (10.21), (10.19), (10.12), and (10.13) for all $(t, x) \in \operatorname{graph}(A)$,

$$
\begin{aligned}
V(t) & \subset\left\{u \in \mathbb{R}^{N}: d_{H}\left(u, U_{r_{2}}(t, x)\right) \leq \gamma\right\} \\
& \subset\left\{u \in \mathbb{R}^{N}: d_{H}(u, U(t, x)) \leq r_{0}\right\} \subset U_{r_{0}}(t, x)
\end{aligned}
$$

and for $i=1,2$,

$$
D_{i} \subset\left\{x \in \mathbb{R}^{n}: d_{H}\left(x, C_{r_{2}}\right) \leq \gamma\right\} \subset\left\{x \in \mathbb{R}^{n}: d_{H}\left(x, C_{i}\right) \leq r_{0}\right\} .
$$

Therefore

$$
S_{D_{1} D_{2} V} \subset S_{C_{r_{0} 1} C_{r_{0} 2} U_{r_{0}}} .
$$

We will show that $(\bar{x}, \bar{u}) \in S_{D_{1} D_{2} V}$. It is sufficient to show that

$$
\bar{x}\left(T_{1}\right) \in D_{1}, \quad \bar{x}\left(T_{2}\right) \in D_{2}
$$

and that for a.e. $t \in\left(T_{1}, T_{2}\right)$,

$$
(t, \bar{x}(t), \bar{u}(t)) \in V(t, x(t)) .
$$

By (10.16)

$$
\bar{x}\left(T_{1}\right) \in C_{r_{1} 1}, \quad \bar{x}\left(T_{2}\right) \in C_{r_{1} 2} .
$$


Let $e_{1}^{n}=(1, \ldots, 0), \ldots, e_{n}^{n}=(0, \ldots, 1)$ be a standard basis in $\mathbb{R}^{n}$ and let $e_{1}^{N}=(1, \ldots, 0), \ldots$, $e_{N}^{N}=(0, \ldots, 1)$ be a standard basis in $\mathbb{R}^{N}$. It follows from $(10.29),(10.13),(10.23),(10.22)$, and (10.21) that for $i=1, \ldots, n, k=1,2$,

$$
\bar{x}\left(T_{k}\right)+2^{-1}\left(r_{0}-r_{1}\right) e_{i}^{n}, \bar{x}\left(T_{k}\right)-2^{-1}\left(r_{0}-r_{1}\right) e_{i}^{n} \in C_{r_{2} k}
$$

and there are $z_{i k}^{(1)}, z_{i k}^{(2)} \in \mathbb{R}^{n}$ such that

$$
\begin{gathered}
\bar{x}\left(T_{k}\right)+z_{i k}^{(1)}, \bar{x}\left(T_{k}\right)+z_{i k}^{(2)} \in D_{k}, \\
\left|z_{i k}^{(1)}-2^{-1}\left(r_{0}-r_{1}\right) e_{i}^{n}\right|,\left|z_{i k}^{(2)}+2^{-1}\left(r_{0}-r_{1}\right) e_{i}^{n}\right| \leq \gamma .
\end{gathered}
$$

Since $D_{k}, k=1,2$ are convex, it follows from these relations, (10.21), and Proposition 8.1 that

$$
0 \in \operatorname{conv}\left\{z_{i k}^{(1)}, z_{i k}^{(2)}: i=1, \ldots, n\right\}, \quad k=1,2
$$

and therefore (10.27) holds.

Assume that $t \in\left(T_{1}, T_{2}\right)$ and

$$
(t, \bar{x}(t), \bar{u}(t)) \in U_{r_{1}}(t, \bar{x}(t)) .
$$

It follows from (10.33), (10.19), (10.12), (10.23), and (10.22) that for $i=1, \ldots, N$,

$$
\bar{u}(t)+2^{-1}\left(r_{0}-r_{1}\right) e_{i}^{n}, \bar{u}(t)-2^{-1}\left(r_{0}-r_{1}\right) e_{i}^{n} \in U_{r_{2}}(t, \bar{x}(t))
$$

and there are $z_{i 1}, z_{i 2} \in \mathbb{R}^{N}$ such that

$$
\begin{gathered}
\bar{u}(t)+z_{i 1}, \bar{u}(t)+z_{i 2} \in V(t, \bar{x}(t)), \\
\left|z_{i 1}-2^{-1}\left(r_{0}-r_{1}\right) e_{i}^{n}\right|,\left|z_{i 2}+2^{-1}\left(r_{0}-r_{1}\right) e_{i}^{n}\right| \leq \gamma .
\end{gathered}
$$

Since $V(t, \bar{x}(t))$ is convex, it follows from these relations, (10.21), and Proposition 8.1 that

$$
0 \in \operatorname{conv}\left\{z_{i 1}, z_{i 2}: i=1, \ldots, N\right\}
$$

and $\bar{u}(t) \in V(t, \bar{x}(t))$. This completes the proof of both the lemma and Theorem 10.1.

\section{A class of nonconvex optimal control problems}

In this section, we again use the notation and definitions from Sections 4 and 5 . Let $m, n, N \geq 1$ be integers, $B_{1}$ and $B_{2}$ fixed nonempty closed subsets of $\mathbb{R}^{n}, \Omega$ a fixed bounded domain in $\mathbb{R}^{m}, H(t, x, u)$ be a fixed continuous function defined on $\Omega \times \mathbb{R}^{n} \times \mathbb{R}^{N}$ with values in $\mathbb{R}^{m n}$ such that $H(t, x, u)=\left(H_{i}\right)_{i=1}^{n}$ and $H_{i}=\left(H_{i j}\right)_{j=1}^{m}, i=1, \ldots, n$, and $\theta^{*}=\left(\theta_{i}^{*}\right)_{i=1}^{n} \in$ $\left(W^{1,1}(\Omega)\right)^{n}$ also fixed. 
Consider a fixed set-valued mapping $A: \Omega \rightarrow 2^{\mathbb{R}^{n}} \backslash\{\varnothing\}$ for which $\operatorname{graph}(A)$ is a closed subset of the space $\Omega \times \mathbb{R}^{n}$ with the product topology and a set-valued mapping $\tilde{U}$ : $\operatorname{graph}(A) \rightarrow 2^{\mathbb{R}^{N}}$ defined by

$$
\tilde{U}(t, x)=\mathbb{R}^{N}, \quad(t, x) \in \operatorname{graph}(A),
$$

(see (4.17)).

We consider the metric space $X\left(A, \widetilde{U}_{A}\right)$, the uniform space $\mathscr{P}_{A}$, the space of integrands $M\left(A, \tilde{U}_{A}\right)$ and its subspaces introduced in Section 4 . Note that all these spaces are equipped with corresponding uniformities and topologies introduced in Section 4.

Denote by $\mathfrak{M}$ the set of all functions $f: \operatorname{graph}(A) \times \mathbb{R}^{N} \rightarrow \mathbb{R}^{1} \cup\{\infty\}$ with the following properties:

(a) $f$ is measurable with respect to the $\sigma$-algebra generated by products of Lebesgue measurable subsets of $\Omega$ and Borel subsets of $\mathbb{R}^{n} \times \mathbb{R}^{N}$;

(b) $f(t, \cdot, \cdot)$ is lower semicontinuous for a.e. $t \in \Omega$;

(c) there exists an integrable scalar function $\psi(t) \leq 0, t \in \Omega$, such that $f(t, x, u) \geq$ $\psi(t)$ for all $(t, x, u) \in \operatorname{graph}(A) \times \mathbb{R}^{N}$.

Property (c) implies that for each $f \in \mathfrak{M}$ and each $(x, u) \in X\left(A, \tilde{U}_{A}\right)$ the function $f(t$, $x(t), u(t)), t \in \Omega$, is measurable.

For the space $\mathfrak{M}$, we consider the uniformity determined by the following base:

$$
E_{\mathfrak{M}}(\epsilon)=\left\{(f, g) \in \mathfrak{M} \times \mathfrak{M}:|f(t, x, u)-g(t, x, u)| \leq \epsilon,(t, x, u) \in \operatorname{graph}(A) \times \mathbb{R}^{N}\right\},
$$

where $\epsilon>0$. It is easy to see that the uniform space $\mathfrak{M}$ with this uniformity is metrizable (by a metric $d_{\mathfrak{M}}$ ) and complete. This uniformity generates in $\mathfrak{M}$ the strong topology. Denote by $\mathfrak{M}_{l}$ (resp., $\mathfrak{M}_{c}$ ) the set of all lower semicontinuous (resp., finite-valued continuous) functions $f: \operatorname{graph}(A) \times \mathbb{R}^{N} \rightarrow \mathbb{R}^{1} \cup\{\infty\}$. Clearly $\mathfrak{M}_{l}$ and $\mathfrak{M}_{c}$ are closed subsets of $\mathfrak{M}$ with the strong topology. It is easy to see that $\mathcal{M}\left(A, \tilde{U}_{A}\right)$ is a closed subset of $\mathfrak{M}$ with the strong topology.

For each $\epsilon>0$, we set

$$
\begin{aligned}
E_{\mathfrak{M}_{w}}(\epsilon)= & \left\{(f, g) \in \mathfrak{M} \times \mathfrak{M}: \text { there exists a nonnegative } \phi \in L^{1}(\Omega)\right. \text { such that } \\
& \int_{\Omega} \phi(t) d t \leq 1 \text { and for a.e. } t \in \Omega,|f(t, x, u)-g(t, x, u)|<\epsilon \\
& \left.+\epsilon \max \{|f(t, x, u)|,|g(t, x, u)|\}+\epsilon \phi(t) \forall x \in A(t), \text { each } u \in \mathbb{R}^{N}\right\} .
\end{aligned}
$$

Using Lemma 4.1 we can easily show that for the set $\mathfrak{M}$ there exists the uniformity which is determined by the base $E_{\mathfrak{M} \epsilon}(\epsilon), \epsilon>0$. This uniformity induces in $\mathfrak{M}$ the weak topology.

Analogously to Proposition 6.1 we can prove the following result.

Proposition 11.1. Let $f \in \mathfrak{M},(x, u) \in X\left(A, \tilde{U}_{A}\right),\left\{\left(x_{i}, u_{i}\right)\right\}_{i=1}^{\infty} \subset X\left(A, \tilde{U}_{A}\right)$, and let $\rho\left(\left(x_{i}\right.\right.$, $\left.\left.u_{i}\right),(x, u)\right) \rightarrow 0$ as $i \rightarrow \infty$. Then

$$
\int_{\Omega} f(t, x(t), u(t)) d t \leq \liminf _{i \rightarrow \infty} \int_{\Omega} f\left(t, x_{i}(t), u_{i}(t)\right) d t .
$$


Analogously to Proposition 6.5 we can prove the following result.

Proposition 11.2. Let $f \in \mathfrak{M}$ and $\epsilon \in(0,1), D>0$. Then there exists a neighborhood $\mathscr{V}$ of $f$ in $\mathfrak{M}$ with the weak topology such that for each $g \in \mathscr{V}$ and each $(x, u) \in X\left(A, \tilde{U}_{A}\right)$ satisfying

$$
\min \left\{\int_{\Omega} f(t, x(t), u(t)) d t, \int_{\Omega} g(t, x(t), u(t)) d t\right\} \leq D,
$$

the following relation holds:

$$
\left|\int_{\Omega} f(t, x(t), u(t)) d t-\int_{\Omega} g(t, x(t), u(t)) d t\right| \leq \epsilon
$$

Denote by $\mathscr{Y}$ the of all functions $\xi: \operatorname{graph}(A) \rightarrow(-\infty, \infty]$ such that for a.e. $t \in \Omega$ the function $\xi(t, \cdot): A(t) \rightarrow(-\infty, \infty]$ is lower semicontinuous. For the set $\mathscr{K}$, we consider the uniformity determined by the following base:

$$
E_{\mathscr{K}}(\epsilon)=\{(\xi, \eta) \in \mathscr{K} \times \mathscr{K}:|\xi(t, x)-\eta(t, x)| \leq \epsilon \forall(t, x) \in \operatorname{graph}(A)\},
$$

where $\epsilon>0$. It is easy to see that the space $\mathscr{K}$ with this uniformity is metrizable (by a metric $\left.d_{\%}\right)$ and complete. This uniformity generates in $\mathscr{Y}$ the strong topology.

For each $\epsilon>0$, we set

$$
\begin{aligned}
E_{\mathscr{K} W}(\epsilon)=\{ & \left(\xi_{1}, \xi_{2}\right) \in \mathscr{K} \times \mathscr{K}:\left|\xi_{1}(t, x)-\xi_{2}(t, x)\right|<\epsilon \\
& \left.+\epsilon \max \left\{\left|\xi_{1}(t, x)\right|,\left|\xi_{2}(t, x)\right|\right\}, x \in A(t)\right\},
\end{aligned}
$$

where $\epsilon>0$. Using Lemma 4.1 we can easily show that for the set $\mathscr{K}$ there exists the uniformity which is determined by the base $E_{\mathfrak{M}_{w}}(\epsilon), \epsilon>0$. This uniformity induces in $\mathscr{K}$ the weak topology. Denote by $\mathscr{K}^{l}$ (resp., $\mathscr{K}^{c}$ ) the set of all lower semicontinuous (resp., finitevalued continuous) functions $\xi \in \mathscr{K}$. Clearly $\mathscr{K}^{l}$ and $\mathscr{K}^{c}$ are closed subsets of $\mathscr{K}$ with the strong topology.

Let $k \geq 1$ be an integer. In the case $m=1$ for each $(f, \xi) \in M\left(A, \tilde{U}_{A}\right) \times C_{l}\left(B_{1}, B_{2}\right)$, each $U \in \mathscr{P}_{A}$, each $\psi_{1}, \ldots, \psi_{k} \in \mathfrak{M}$, and each $\psi \in \mathscr{K}$, we consider the optimal control problem

$$
\begin{gathered}
I^{(f, \xi)}(x, u) \longrightarrow \min , \\
(x, u) \in X(A, U), \quad \psi(t, x(t)) \leq 0, \quad t \in \Omega \text { a.e. } \\
\int_{\Omega} \psi_{i}(t, x(t), u(t)) d t \leq 0, \quad i=1, \ldots, k .
\end{gathered}
$$

In the case $m>1$ for each $f \in \mathcal{M}\left(A, \tilde{U}_{A}\right)$, each $U \in \mathscr{P}_{A}$, each $\psi_{1}, \ldots, \psi_{k} \in \mathfrak{M}$, and each $\psi \in \mathscr{K}$, we consider the optimal control problem

$$
\begin{gathered}
I^{(f)}(x, u) \longrightarrow \min , \\
(x, u) \in X\left(A, \tilde{U}_{A}\right), \quad \psi(t, x(t)) \leq 0, \quad t \in \Omega \text { a.e. } \\
\int_{\Omega} \psi_{i}(t, x(t), u(t)) d t \leq 0, \quad i=1, \ldots, k .
\end{gathered}
$$


Define a space $\mathscr{A}_{1}$ as follows:

$$
\mathscr{A}_{1}=\mathscr{A}_{11} \times \mathscr{A}_{12} \quad \text { if } m=1, \quad \mathscr{A}_{1}=\mathscr{A}_{11} \quad \text { if } m>1,
$$

where $\mathscr{A}_{12}$ is either $C_{l}\left(B_{1} \times B_{2}\right)$ or $C\left(B_{1} \times B_{2}\right)$ or a singleton $\{\xi\} \subset C_{l}\left(B_{1} \times B_{2}\right)$, and $\mathscr{A}_{11}$ is one of the following spaces:

$$
\begin{gathered}
M\left(A, \tilde{U}_{A}\right) ; \quad M^{l}\left(A, \tilde{U}_{A}\right) ; \quad M^{c}\left(A, \tilde{U}_{A}\right) ; \\
\mathcal{M}_{k}\left(A, \tilde{U}_{A}\right) ; \quad M_{k}^{l}\left(A, \tilde{U}_{A}\right) ; \quad M_{k}^{c}\left(A, \tilde{U}_{A}\right) \quad \text { (here } k \geq 1 \text { is an integer); } \\
\left.\mathcal{M}_{k}^{*}(\tilde{A}, \tilde{U}) ; \quad M_{k}^{* l}(\tilde{A}, \tilde{U}) ; \quad M_{k}^{* c}(\tilde{A}, \tilde{U}) \quad \text { (here } k \geq 1 \text { is an integer and } A=\tilde{A}\right) .
\end{gathered}
$$

Define a space $\mathscr{A}_{2}$ as follows:

$$
\mathscr{A}_{2}=\mathscr{A}_{20} \times \mathscr{A}_{21} \times \cdots \times \mathscr{A}_{2 k} \times \mathscr{P}_{A}
$$

where $\mathscr{A}_{20}$ is either $\mathscr{K}_{\mathcal{L}}$ or $\mathscr{K}^{l}$ or $\mathscr{K}^{c}$ and $\mathscr{A}_{2 i}(i=1, \ldots, k)$ is either $\mathfrak{M}$ or $\mathfrak{M}_{l}$ or $\mathfrak{M}_{c}$ or a singleton $\{\xi\} \in \mathfrak{M}$.

For each $a=\left(a_{0}, \ldots, a_{k}, U\right) \in \mathscr{A}_{2}$, define

$$
\begin{gathered}
S_{a_{2}}=\left\{(x, u) \in X(A, U): a_{0}(t, x(t)) \leq 0 t \in \Omega\right. \text { a.e. and } \\
\left.\int_{\Omega} a_{i}(t, x(t), u(t)) d t \leq 0, i=1, \ldots, k\right\} .
\end{gathered}
$$

For each $a=\left(a_{1}, a_{2}\right) \in \mathscr{A}$, we define $J_{a}: X\left(A, \tilde{U}_{A}\right) \rightarrow \mathbb{R}^{1} \cup\{\infty\}$ by

$$
\begin{gathered}
J_{a}(x, u)=I^{\left(a_{1}\right)}(x, u):(x, u) \in S_{a_{2}}, \\
J_{a}(x, u)=\infty, \quad(x, u) \in X\left(A, \tilde{U}_{A}\right) \backslash S_{a_{2}} .
\end{gathered}
$$

By Propositions 6.1, 6.2, and $11.1 J_{a}$ is lower semicontinuous function for all $a \in \mathscr{A}_{1} \times \mathscr{A}_{2}$.

Denote by $\mathscr{A}$ the closure of the set $\left\{a \in \mathscr{A}_{1} \times \mathscr{A}_{2}: \inf \left(J_{a}\right)<\infty\right\}$ in the space $\mathscr{A}_{1} \times A_{2}$ with the strong topology. We assume that $\mathscr{A}$ is nonempty. We will establish the following result.

Theorem 11.3. There exists an everywhere dense (in the strong topology) set $\mathscr{B} \subset \mathscr{A}$ which is a countable intersection of open (in the weak topology) subsets of $\mathscr{A}$ such that for any $a \in \mathscr{B}$, the following assertions hold:

(1) $\inf \left(J_{a}\right)$ is finite and attained at a unique pair $(\bar{x}, \bar{u}) \in X\left(A, \tilde{U}_{A}\right)$,

(2) for each $\epsilon>0$, there are a neighborhood $\mathscr{V}$ of $a$ in $\mathscr{A}$ with the weak topology and $\delta>0$ such that for each $b \in \mathscr{V}, \inf \left(J_{b}\right)$ is finite and if $(z, w) \in X\left(A, \tilde{U}_{A}\right)$ satisfies $J_{b}(z, w) \leq \inf \left(J_{b}\right)+\delta$, then $\rho((\bar{x}, \bar{u}),(z, w)) \leq \epsilon$ and $\left|J_{b}(z, w)-J_{a}(\bar{x}, \bar{u})\right| \leq \epsilon$.

Proof. By Propositions 6.1, 6.2, and 11.1 (A1) holds and $J_{a}$ is lower semicontinuous for all $a \in \mathscr{A}_{1} \times \mathscr{A}_{2}$. By Theorem 5.1 we need to verify that (H1) and (H2) are valid. Hypothesis (H2) follows from Proposition 6.2. Therefore it is sufficient to show that (H1) holds. By Proposition 5.3 it is sufficient to show that (A2), (A3), and (A4) are valid. Hypothesis (A2) follows from Propositions 6.5 and 6.7. By Lemma 7.1, (A3) holds. Hypothesis (A4) will follow from Lemma 11.4 below. 
Recall that $e_{1}=(1, \ldots, 0), \ldots, e_{p}=(0, \ldots, 1)$ is the standard basis in $\mathbb{R}^{p}$. In the case $m=1$ we set $\mathscr{L}=C_{l}\left(B_{1} \times B_{2}\right)$ and in the case $m>1$ we denote by $\mathscr{L}$ a singleton $\{0\}$ where 0 is a function in $C_{l}\left(B_{1} \times B_{2}\right)$ which is identically zero. In the case $m>1$ for each $(f, \xi) \in M\left(A, \tilde{U}_{A}\right) \times \mathscr{L}$ and each $(x, u) \in X\left(A, \tilde{U}_{A}\right)$, we set

$$
I^{(f, \xi)}(x, u)=I^{f}(x, u) \quad(\text { see }(4.15) \text { and }(4.16)) .
$$

Lemma 11.4. Let $f \in M\left(A, \tilde{U}_{A}\right), \xi \in \mathscr{L}, U \in \mathscr{P}_{A}$,

$$
\begin{gathered}
\psi_{i} \in \mathscr{A}_{2 i}, \quad i=0, \ldots, k, \quad a_{2}=\left(\psi_{0}, \ldots, \psi_{k}, U\right), \\
\left\{(x, u) \in S_{a_{2}}: I^{(f, \xi)}(x, u)<\infty\right\} \neq \varnothing
\end{gathered}
$$

and let $\epsilon, \delta>0$. Then there are

$$
\left(a_{* 2}\right)=\left(\psi_{* 0}, \ldots, \psi_{* k}, U_{*}\right),
$$

where

$$
U_{*} \in \mathscr{P}_{A}, \quad \psi_{* i} \in \mathscr{A}_{2 i}, \quad i=0, \ldots, k, \quad(\bar{x}, \bar{u}) \in S_{a_{*} 2},
$$

and a nonempty open subset $\mathcal{W}$ of $\mathscr{A}_{2}$ with the weak topology such that

$$
\begin{gathered}
\left(U_{*}, U\right) \in E_{\mathscr{P}_{A}}(\epsilon), \quad\left(\psi_{* 0}, \psi_{0}\right) \in E_{\mathscr{K}}(\epsilon), \\
\left(\psi_{i}, \psi_{* i}\right) \in E_{\mathfrak{M}}(\epsilon), \quad i=1, \ldots, k, \\
\mathcal{W} \cap\left\{\left(\phi_{0}, \ldots, \phi_{k}, V\right) \in \mathscr{A}_{2}:(U, V) \in \mathscr{P}_{A}(\epsilon),\right. \\
\left.\left(\phi_{i}, \psi_{i}\right) \in E_{\mathfrak{M}}(\epsilon), i=1, \ldots, k,\left(\phi_{0}, \psi_{0}\right) \in E_{\mathscr{K}}(\epsilon)\right\} \neq \varnothing, \\
I^{(f, \xi)}(\bar{x}, \bar{u}) \leq \inf \left\{I^{(f, \xi)}(x, u):(x, u) \in S_{a_{* 2}}\right\}+\delta<\infty
\end{gathered}
$$

and for all $b_{2} \in \mathcal{W}$,

$$
(\bar{x}, \bar{u}) \in S_{b_{2}} \subset S_{a_{* 2}} .
$$

Proof. For each $r \in(0,1]$, define $U_{r} \in \mathscr{P}_{A}$ by

$$
U_{r}(t, x)=\left\{u \in \mathbb{R}^{n}: d_{H}(u, U(t, x)) \leq r\right\}, \quad(t, x) \in \operatorname{graph}(A),
$$

define $\psi_{r i} \in \mathscr{A}_{2 i}, i=0, \ldots, k$ by

$$
\begin{gathered}
\psi_{r 0}(t, x)=\psi_{0}(t, x)-r, \quad(t, x) \in \operatorname{graph}(A), \\
\psi_{r i}(t, x, u)=\psi_{i}(t, x, u)-r, \quad(t, x, u) \in \operatorname{graph}(A) \times \mathbb{R}^{N}, i=1, \ldots, k .
\end{gathered}
$$

Set

$$
a_{r 2}=\left(\psi_{r 0}, \psi_{r 1}, \ldots, \psi_{r k}, U_{r}\right)
$$


406 Solutions of optimal control problems

and put

$$
\mu(r)=\inf \left\{I^{(f, \xi)}(x, u):(x, u) \in S_{a_{r 2}}\right\} .
$$

Clearly $\mu(r)$ is finite for all $r \in(0,1]$ and the function $\mu$ is monotone decreasing. There is $r_{0} \in\left(0, \min \left\{8^{-1}, 8^{-1} \epsilon\right\}\right)$ such that $\mu$ is continuous at $r_{0}$. Choose $r_{1} \in\left(0, r_{0}\right]$ such that

$$
\left|\mu\left(r_{1}\right)-\mu\left(r_{0}\right)\right|<16^{-1} \delta .
$$

There is

$$
(\bar{x}, \bar{u}) \in S_{a_{r_{1} 2}}
$$

such that

$$
I^{(f, \bar{\xi})}(\bar{x}, \bar{u}) \leq \mu\left(r_{1}\right)+16^{-1} \delta .
$$

Relations (11.30), (11.32) imply that

$$
I^{(f, \bar{\xi})}(\bar{x}, \bar{u}) \leq \mu\left(r_{0}\right)+8^{-1} \delta .
$$

Set

$$
r_{2}=2^{-1}\left(r_{0}+r_{1}\right)
$$

Clearly

$$
\begin{gathered}
\left(U_{r_{i}}, U\right) \in E_{\mathscr{P}_{A}}(\epsilon), \quad i=0,1,2, \\
\left(\psi_{r_{i} 0}, \psi_{0}\right) \in E_{\mathscr{Y}}(\epsilon), \quad i=0,1,2, \\
\left(\psi_{r_{i} j}, \psi_{j}\right) \in E_{\mathfrak{M}}(\epsilon), \quad i=0,1,2, j=1, \ldots, k
\end{gathered}
$$

In view of property (c) (see the definition of $\mathfrak{M}$ ), there exists an integrable scalar function $\bar{\phi}(t) \geq 0, t \in \Omega$ such that

$$
\psi_{i}(t, x, u) \geq-\bar{\phi}(t) \quad \forall(t, x, u) \in \operatorname{graph}(A) \times \mathbb{R}^{N} \text { and all } i=1, \ldots, k
$$

Choose a positive number $\gamma_{0}$ such that

$$
\begin{gathered}
\gamma_{0}<\min \left\{4^{-1} \delta, 16^{-1},(16 N)^{-1}\left(r_{0}-r_{1}\right)\right\}, \\
\gamma_{0}\left[\operatorname{mes}(\Omega)+1+\int_{\Omega} \bar{\phi}(t) d t\right]<\left(r_{0}-r_{2}\right) \operatorname{mes}(\Omega)
\end{gathered}
$$

and choose a positive number $\gamma<\gamma_{0}$ such that

$$
\gamma+\gamma(1-\gamma)^{-1}<\frac{\gamma_{0}}{8}
$$


Let $\mathcal{W}$ be the interior of the subset

$$
\begin{aligned}
\{\eta \in \mathscr{K} & \left.:\left(\eta, \psi_{r_{2} 0}\right) \in E_{\mathscr{K} w}(\gamma)\right\} \times \prod_{j=1}^{q}\left\{\xi \in \mathscr{A}_{2 j}:\left(\xi, \psi_{r_{2} j}\right) \in E_{\mathfrak{M}_{w}}(\gamma)\right\} \\
& \times\left\{V \in \mathscr{P}_{A}:\left(V, U_{r_{2}}\right) \in E_{\mathscr{P}_{A}}(\gamma)\right\}
\end{aligned}
$$

of $\mathscr{A}_{2}$ with the weak topology. Set

$$
\begin{gathered}
U_{*}=U_{r_{0}}, \quad \psi_{* j}=\psi_{r_{0} j}, \quad j=0, \ldots, k, \\
a_{* 2}=\left(\psi_{* 0}, \ldots, \psi_{* k}, U_{*}\right) .
\end{gathered}
$$

By (11.41), (11.31), (11.34), and (11.28) the inclusions (11.21) hold. The choice of $W$ (see (11.40)), (11.41), (11.35) imply that (11.22) hold. Relations (11.23) follow from (11.33), (11.29), (11.28), and (11.41). In order to complete the proof of the lemma, it is sufficient to show that (11.24) is true for all $b_{2} \in \mathcal{W}$.

Assume that

$$
b_{2}=\left(\xi_{0}, \xi_{1}, \ldots, \xi_{k}, V\right) \in \mathcal{W}
$$

where

$$
\xi_{j} \in \mathscr{A}_{2 j}, \quad j=0, \ldots, k, \quad V \in \mathscr{P}_{A} .
$$

It follows from (11.43), (11.44), the choice of $\mathcal{W}$ (see (11.40)), (11.25), (11.37), (11.39), and (11.34) that for each $(t, x) \in \operatorname{graph}(A)$,

$$
\begin{aligned}
V(t, x) & \subset\left\{z \in \mathbb{R}^{N}: d_{H}\left(z, U_{r_{2}}(t, x)\right) \leq \gamma\right\} \\
& \subset\left\{z \in \mathbb{R}^{N}: d_{H}(z, U(t, x)) \leq r_{0}\right\}=U_{r_{0}}(t, x), \\
V(t, x) & \subset U_{r_{0}}(t, x) .
\end{aligned}
$$

By (11.43), (11.44), the choice of $\mathcal{W}($ see $(11.40)),(11.8)$ for a.e. $t \in \Omega$

$$
\left|\xi_{0}(t, x)-\psi_{r_{2} 0}(t, x)\right|<\gamma+\gamma \max \left\{\left|\xi_{0}(t, x)\right|,\left|\psi_{r_{2} 0}(t, x)\right|\right\} \quad \forall x \in A(t) .
$$

Relations (11.47) and (11.39) and Lemma 4.1 imply that for a.e. $t \in \Omega$

$$
\left|\xi_{0}(t, x)-\psi_{r_{2} 0}(t, x)\right|<8^{-1} \gamma_{0}+8^{-1} \gamma_{0} \min \left\{\left|\xi_{0}(t, x)\right|,\left|\psi_{r_{2} 0}(t, x)\right|\right\} \quad \forall x \in A(t) .
$$

We show that the following implication holds.

(i1) for a.e. $t$, if $y \in A(t)$ satisfies $\xi_{0}(t, y) \leq 0$, then

$$
\psi_{r_{0} 0}(t, y) \leq 0
$$


408

Solutions of optimal control problems

Assume that $t \in \Omega$, (11.48) holds, $y \in A(t)$, and

$$
\xi_{0}(t, y) \leq 0
$$

We show that $\psi_{r_{0} 0}(t, y) \leq 0$. We assume the converse. Then $\psi_{r_{0} 0}(t, y)>0$. It follows from this inequality, (11.27), and (11.34) that

$$
\begin{aligned}
\psi_{r_{2} 0}(t, y) & =\psi_{0}(t, y)-r_{2}=\psi_{0}(t, y)-r_{0}+r_{0}-r_{2} \\
& =\psi_{r_{0} 0}(t, y)+\frac{r_{0}-r_{1}}{2}>\frac{r_{0}-r_{1}}{2} .
\end{aligned}
$$

Combined with (11.48) and (11.37), relation (11.51) implies that

$$
\begin{aligned}
\xi_{0}(t, y) & \geq \psi_{r_{2} 0}(t, y)-8^{-1} \gamma_{0}-8^{-1} \gamma_{0} \psi_{r_{2} 0}(t, y) \\
& \geq-8^{-1} \gamma_{0}+\psi_{r_{2} 0}(t, y)\left(1-8^{-1} \gamma_{0}\right) \\
& \geq-8^{-1} \gamma_{0}+\frac{r_{0}-r_{1}}{4}>0
\end{aligned}
$$

a contradiction. The contradiction we have reached proves the inequality $\psi_{r_{2} 0}(t, y) \leq 0$ and implication (i1).

Now we show that the following implication holds:

(i2) for each $j \in\{1, \ldots, k\}$ and each $(x, u) \in X\left(A, \tilde{U}_{A}\right)$,

$$
\begin{aligned}
\int_{\Omega} \mid \xi_{j}( & t, x(t), u(t))-\psi_{r_{2} j}(t, x(t), u(t)) \mid \\
\leq & \frac{3}{4} \gamma_{0} \operatorname{mes}(\Omega)+4^{-1} \gamma_{0}+2^{-1} \gamma_{0} \int_{\Omega} \bar{\phi}(t) d t \\
& +8^{-1} \gamma_{0} \int_{\Omega} \min \left\{\xi_{j}(t, x(t), u(t)), \psi_{r_{2} j}(t, x(t), u(t))\right\} d t .
\end{aligned}
$$

Assume that $j \in\{1, \ldots, k\},(x, u) \in X\left(A, \tilde{U}_{A}\right)$. It follows from (11.43), (11.44), the choice of $\mathcal{W}($ see $(11.40))$ and (11.3) that there exists a nonnegative $\phi_{j} \in L^{1}(\Omega)$ such that

$$
\int_{\Omega} \phi_{j}(t) d t \leq 1
$$

and for a.e. $t \in \Omega$,

$$
\begin{array}{r}
\left|\xi_{j}(t, y, v)-\psi_{r_{2} j}(t, y, v)\right|<\gamma+\gamma \max \left\{\left|\xi_{j}(t, y, v)\right|,\left|\psi_{r_{2} j}(t, y, v)\right|\right\}+\gamma \phi_{j}(t) \\
\text { for each } y \in A(t) \text { each } v \in \mathbb{R}^{N} .
\end{array}
$$

By (11.55), (11.39), and Lemma 4.1 for a.e. $t \in \Omega$,

$$
\left|\xi_{j}(t, y, v)-\psi_{r_{2} j}(t, y, v)\right|<\left(1+\phi_{j}(t)\right) 8^{-1} \gamma_{0}+8^{-1} \gamma_{0} \min \left\{\left|\xi_{j}(t, y, v)\right|,\left|\psi_{r_{2} j}(t, y, v)\right|\right\}
$$

for each $y \in A(t)$ and each $v \in \mathbb{R}^{N}$. 
In view of (11.56), (11.27), and (11.36) for a.e. $t \in \Omega$,

$$
\begin{aligned}
\xi_{j}(t, y, v) & \geq \psi_{r_{2} j}(t, y, v)-8^{-1} \gamma_{0}\left(1+\phi_{j}(t)\right)-8^{-1} \gamma_{0}\left|\psi_{r_{2} j}(t, y, v)\right| \\
& \geq-8^{-1} \gamma_{0}\left(1+\phi_{j}(t)\right)+\psi_{j}(t, y, v)-r_{2}-2^{-1} \gamma_{0}\left|\psi_{j}(t, y, v)\right|-r_{2} \\
& \geq-8^{-1} \gamma_{0}\left(1+\phi_{j}(t)\right)-\bar{\phi}(t)-2
\end{aligned}
$$

for each $y \in A(t)$ and each $v \in \mathbb{R}^{N}$.

It follows from (11.56), (11.57), (11.27), and (11.36) that for a.e. $t \in \mathbb{R}^{1}$,

$$
\begin{gathered}
\left|\xi_{j}(t, y, v)-\psi_{r_{2} j}(t, y, v)\right| \\
<\left(1+\phi_{j}(t)\right) 8^{-1} \gamma_{0}+8^{-1} \gamma_{0} \min \left\{\xi_{j}(t, y, v)+4^{-1} \gamma_{0}\left(1+\phi_{j}(t)\right)+2 \bar{\phi}(t)\right. \\
\left.+4,1+\psi_{r_{2} j}(t, y, v)+2 \bar{\phi}(t)\right\} \\
\leq 8^{-1} \gamma_{0}\left(1+\phi_{j}(t)\right)+8^{-1} \gamma_{0} \min \left\{\xi_{j}(t, y, v), \psi_{r_{2} j}(t, y, v)\right\} \\
+8^{-1} \gamma_{0}\left[4^{-1} \gamma_{0}\left(1+\phi_{j}(t)\right)+2 \bar{\phi}(t)+4\right]
\end{gathered}
$$

for each $y \in A(t)$, each $v \in \mathbb{R}^{N}$.

By (11.58) and (11.54),

$$
\begin{aligned}
\int_{\Omega} \mid \xi_{j}( & t, x(t), u(t))-\psi_{r_{2} j}(t, x(t), u(t)) \mid d t \\
\leq & 8^{-1} \gamma_{0} \int_{\Omega}\left(1+\phi_{j}(t)\right) d t+8^{-1} \gamma_{0} \int_{\Omega} \min \left\{\xi_{j}(t, x(t), u(t)), \psi_{r_{2} j}(t, x(t), u(t))\right\} d t \\
& +32^{-1} \gamma_{0}^{2} \int_{\Omega}\left[1+\phi_{j}(t)\right] d t+8^{-1} \gamma_{0} \int_{\Omega}[2 \bar{\phi}(t) d t+4] d t \\
\leq & 8^{-1} \gamma_{0} \operatorname{mes}(\Omega)+8^{-1} \gamma_{0}+32^{-1} \gamma_{0}^{2} \operatorname{mes}(\Omega)+32^{-1} \gamma_{0}^{2}+2^{-1} \gamma_{0} \operatorname{mes}(\Omega) \\
& +4^{-1} \gamma_{0} \int_{\Omega} \bar{\phi}(t) d t+8^{-1} \gamma_{0} \int_{\Omega} \min \left\{\xi_{j}(t, x(t), u(t)), \psi_{r_{2} j}(t, x(t), u(t))\right] d t .
\end{aligned}
$$

In view of (11.59), implication (i2) is true.

Assume that

$$
(x, u) \in S_{b_{2}} .
$$

It follows from (11.60), (11.43), (11.44), and (11.14) that for a.e. $t \in \Omega$,

$$
\xi_{0}(t, x(t)) \leq 0
$$

Combined with (i1), this implies that for a.e. $t \in \Omega$,

$$
\psi_{r_{0}}(t, x(t)) \leq 0 \text {. }
$$

Let $j \in\{1, \ldots, k\}$. By (11.60), (11.43), (11.44), and (11.14),

$$
\int_{\Omega} \xi_{j}(t, x(t), u(t)) d t \leq 0 .
$$


410 Solutions of optimal control problems

(11.63) and (i2) imply that

$$
\begin{aligned}
& \int_{\Omega} \psi_{r_{2} j}(t, x(t), u(t)) d t \\
& \leq \int_{\Omega} \xi_{j}(t, x(t), u(t)) d t+\frac{3}{4} \gamma_{0} \operatorname{mes}(\Omega)+4^{-1} \gamma_{0} \\
& \quad+2^{-1} \gamma_{0} \int_{\Omega} \bar{\phi}(t) d t+2^{-1} \gamma_{0} \int_{\Omega} \xi_{j}(t, x(t), u(t)) d t \\
& \leq \frac{3}{4} \gamma_{0} \operatorname{mes}(\Omega)+4^{-1} \gamma_{0}+2^{-1} \gamma_{0} \int_{\Omega} \bar{\phi}(t) d t
\end{aligned}
$$

In view of (11.64), (11.27), and (11.37),

$$
\begin{aligned}
\int_{\Omega} \psi_{r_{0} j}(t, x(t), u(t)) d t & =\int_{\Omega}\left[\psi_{r_{2} j}(t, x(t), u(t))-\left(r_{0}-r_{2}\right)\right] d t \\
& =-\left(r_{0}-r_{2}\right) \operatorname{mes}(\Omega)+\int_{\Omega} \psi_{r_{2} j}(t, x(t), u(t)) d t \\
& \leq-\left(r_{0}-r_{2}\right) \operatorname{mes}(\Omega)+\gamma_{0}\left[\operatorname{mes}(\Omega)+1+\int_{\Omega} \phi(t) d t\right]<0 .
\end{aligned}
$$

Thus

$$
\int_{\Omega} \psi_{r_{0} j}(t, x(t), u(t)) \leq 0, \quad j=1, \ldots, k
$$

It follows from (11.60), (11.43), (11.44), and (11.14) that for a.e. $t \in \Omega, u(t) \in V(t, x(t))$. Combined with (11.46) this implies that for a.e. $t \in \Omega, u(t) \in U_{r_{0}}(t, x(t))$. Combined with (11.66), (11.62), (11.43), (11.44), (11.14), and (11.41) this implies that

$$
(x, u) \in S_{a_{*} 2}=S_{a_{r_{0}}} .
$$

Therefore, we have shown that

$$
S_{b_{2}} \subset S_{a_{* 2}}
$$

Now we show that

$$
(\bar{x}, \bar{u}) \in S_{b_{2}} .
$$

Relations (11.31), (11.28), and (11.14) imply that for a.e. $t \in \Omega$,

$$
\bar{u}(t) \in U_{r_{1}}(t, \bar{x}(t)) .
$$

Assume that $t \in \Omega$ and (11.70) holds. By (11.70), (11.25), and (11.34) for $i=1, \ldots, N$,

$$
\bar{u}(t)+2^{-1}\left(r_{0}-r_{1}\right) e_{i}, \bar{u}(t)+2^{-1}\left(r_{0}-r_{1}\right) e_{i} \in U_{r_{2}}(t, \bar{x}(t))
$$


In view of (11.71), (11.43), (11.44), the choice of $\mathcal{W}$ (see $(11.40))$, for $i=1, \ldots, N$, there are $z_{i 1}, z_{i 2} \in \mathbb{R}^{N}$ such that

$$
\begin{gathered}
\bar{u}(t)+z_{i 1}, \bar{u}(t)+z_{i 2} \in V(t, \bar{x}(t)), \\
\left|z_{i 1}-2^{-1}\left(r_{0}-r_{1}\right) e_{i}\right|,\left|z_{i 2}+2^{-1}\left(r_{0}-r_{1}\right) e_{i}\right| \leq \gamma .
\end{gathered}
$$

Since the set $V(t, \bar{x}(t))$ is convex, from (11.73), (11.37), (11.39) and Proposition 8.1 it follows that

$$
\begin{gathered}
0 \in \operatorname{conv}\left\{z_{i j}: i=1, \ldots, N, j=1,2\right\}, \\
\bar{u}(t) \in V(t, \bar{x}(t)) .
\end{gathered}
$$

Thus we have shown that

$$
\bar{u}(t) \in V(t, \bar{x}(t)) \quad \text { for a.e. } t \in \Omega \text {. }
$$

We show that for a.e. $t \in \Omega, \xi_{0}(t, \bar{x}(t)) \leq 0$.

It follows from (11.31), (11.28), (11.14), (11.18), and (11.34) that for a.e. $t \in \Omega$

$$
\begin{aligned}
0 & \geq \psi_{r_{1} 0}(t, \bar{x}(t))=\psi_{0}(t, \bar{x}(t))-r_{1}, \\
\psi_{r_{2} 0}(t, \bar{x}(t)) & =\psi_{0}(t, \bar{x}(t))-r_{2}=\psi_{0}(t, \bar{x}(t))-r_{1}+r_{1}-r_{2} \\
& =\psi_{r_{1} 0}(t, \bar{x}(t))-\frac{r_{0}-r_{1}}{2} \leq-\frac{r_{0}-r_{1}}{2} .
\end{aligned}
$$

Relations (11.77), (11.48), and (11.37) imply that for a.e. $t \in \Omega$,

$$
\begin{aligned}
\xi_{0}(t, \bar{x}(t)) & \leq \psi_{r_{2} 0}(t, \bar{x}(t))+8^{-1} \gamma_{0}+8^{-1} \gamma_{0} \psi_{r_{2} 0}(t, \bar{x}(t)) \\
& \leq \frac{1}{2} \psi_{r_{2} 0}(t, \bar{x}(t))+8^{-1} \gamma_{0} \\
& \leq-\frac{r_{0}-r_{1}}{4}+8^{-1} \gamma_{0}<0 .
\end{aligned}
$$

Thus we have shown that

$$
\xi_{0}(t, \bar{x}(t)) \leq 0 \quad \text { for a.e. } t \in \Omega \text {. }
$$

Let $j \in\{1, \ldots, k\}$. We show that

$$
\int_{\Omega} \xi_{j}(t, \bar{x}(t), \bar{u}(t)) d t \leq 0
$$

It follows from (11.31), (11.28), and (11.14) that

$$
\int_{\Omega} \psi_{r_{1} j}(t, \bar{x}(t), \bar{u}(t)) d t \leq 0 \text {. }
$$


412 Solutions of optimal control problems

Relations (11.27), (11.34), and (11.81) imply that

$$
\begin{aligned}
\int_{\Omega} \psi_{r_{2} j}(t, \bar{x}(t), \bar{u}(t)) d t \\
\quad=\int_{\Omega}\left[\psi_{j}(t, \bar{x}(t), \bar{u}(t))-r_{2}\right] d t \\
\quad=\int_{\Omega}\left[\left(\psi_{j}(t, \bar{x}(t), \bar{u}(t))-r_{1}\right)+\left(r_{1}-r_{2}\right)\right] d t \\
\quad=\int_{\Omega} \psi_{r_{1} j}(t, \bar{x}(t), \bar{u}(t)) d t-\left(\frac{r_{0}-r_{1}}{2}\right) \operatorname{mes}(\Omega) \\
\quad \leq-\operatorname{mes}(\Omega) \frac{r_{0}-r_{1}}{2} .
\end{aligned}
$$

By implication (i2) (see (11.53)), (11.82), and (11.37),

$$
\begin{aligned}
\int_{\Omega} \xi_{j}(t, \bar{x}(t), \bar{u}(t)) d t \leq & \int_{\Omega} \psi_{r_{2} j}(t, \bar{x}(t), \bar{u}(t)) d t+\frac{3}{4} \gamma_{0} \operatorname{mes}(\Omega)+4^{-1} \gamma_{0} \\
& +2^{-1} \gamma_{0} \int_{\Omega} \bar{\phi}(t) d t+8^{-1} \gamma_{0} \int_{\Omega} \psi_{r_{2} j}(t, \bar{x}(t), \bar{u}(t)) d t \\
\leq & -\operatorname{mes}(\Omega) \frac{r_{0}-r_{1}}{2}+\gamma_{0} \operatorname{mes}(\Omega)+\gamma_{0}+\gamma_{0} \int_{\Omega} \bar{\phi}(t) d t<0 .
\end{aligned}
$$

Therefore, we have shown that

$$
\int_{\Omega} \xi_{j}(t, \bar{x}(t), \bar{u}(t)) d t<0, \quad j=1, \ldots, k
$$

Relations (11.75), (11.79), (11.84), (11.43), and (11.14) imply that $(\bar{x}, \bar{u}) \in S_{b_{2}}$. Thus (11.24) holds for all $b_{2} \in \mathcal{W}$. This completes the proof of the lemma.

\section{Minimization problems with constraints}

In this section, we discuss three classes of minimization problems with constraints. For these classes, generic existence of solutions is obtained as a realization of our variational principle (see Theorem 5.1 and Proposition 5.3).

Let $(X, \rho)$ be a complete metric space and let $C_{l}(X)$ be the set of all lower semicontinuous functions $f: X \rightarrow \mathbb{R}^{1} \cup\{\infty\}$. Denote by $C_{b l}$ the set of all bounded from bellow functions $f \in C_{l}(X)$.

For each function $f: Y \rightarrow[-\infty, \infty]$, where $Y$ is nonempty set, we define

$$
\begin{gathered}
\operatorname{dom}(f)=\{y \in Y:-\infty<f(y)<\infty\}, \\
\inf (f)=\{f(y): y \in Y\}
\end{gathered}
$$

We use the convention that $\infty-\infty=0$.

Denote by $C(X)$ the set of all continuous real-valued functions $f \in C_{l}(X)$ and set $C_{b}(X)=C(X) \cap C_{b l}(X)$. We equip the set $C_{l}(X)$ with a strong and weak topologies. 
For the set $C_{l}(X)$, we consider the uniformity determined by the following base:

$$
\begin{aligned}
E_{C s}(\epsilon)= & \left\{(g, h) \in C_{l}(X) \times C_{l}(X):|g(x)-h(x)| \leq \epsilon \forall x \in X\right. \text { and } \\
& |(g(x)-h(x))-(g(y)-h(y))| \leq \epsilon \rho(x, y) \text { for each } x, y \in \operatorname{dom}(g)\},
\end{aligned}
$$

where $\epsilon>0$. Clearly this uniform space $C_{l}(X)$ is metrizable (by a metric $d_{C s}$ ) and complete. We equip the set $C_{l}(X)$ with the strong topology induced by this uniformity.

Now we equip the set $C_{l}(X)$ with a weak topology. For each $\epsilon>0$, we set

$$
E_{C w}(\epsilon)=\left\{(g, h) \in C_{l}(X) \times C_{l}(X):|g(x)-h(x)|<\epsilon+\epsilon \max \{|g(x)|,|h(x)|\} \forall x \in X\right\} .
$$

We can show in a straightforward manner that for the set $C_{l}(X)$ there exists a uniformity which is determined by the base $E_{C w}(\epsilon), \epsilon>0$. It is easy to see that this uniformity is metrizable (by a metric $d_{C w}$ ) and complete. This uniformity induces on $C_{l}(X)$ the weak topology. Clearly $C(X), C_{b}(X)$, and $C_{b l}(X)$ are closed subsets of $C_{l}(X)$ with the strong topology.

Now we define spaces $\mathscr{A}_{1}$ and $\mathscr{A}_{2}$. Let $\mathscr{A}_{1}$ be either $C_{b l}(X)$ or $C_{b}(X)$ and let $\mathscr{A}_{2}=$ $C_{1}^{*} \times \cdots \times C_{n}^{*}$ where $C_{i}^{*}, i=1, \ldots, n$ is one of the following spaces:

$$
C_{l}(X) ; \quad C(X) ; \quad C_{b l}(X) ; \quad C_{b}(X)
$$

For $a \in \mathscr{A}_{1}$, we set $\phi_{a}=a$ and for $g=\left(g_{1}, \ldots, g_{n}\right) \in \mathscr{A}_{2}$, we set

$$
S_{g}=\left\{x \in X: g_{i}(x) \leq 0, i=1, \ldots, n\right\}
$$

For $a=\left(a_{1}, a_{2}\right) \in \mathscr{A}_{1} \times \mathscr{A}_{2}$, we define a function $f_{a}: X \rightarrow \mathbb{R}^{1} \cup\{\infty\}$ by

$$
f_{a}(x)=\phi_{a_{1}}(x)=a_{1}(x), \quad x \in S_{a_{2}}, \quad f_{a}(x)=\infty, \quad x \in X \backslash S_{a_{2}} .
$$

Denote by $\mathscr{A}$ the closure of the set $\left\{a \in \mathscr{A}_{1} \times \mathscr{A}_{2}: \inf \left(f_{a}\right)<\infty\right\}$ in the space $\mathscr{A}_{1} \times \mathscr{A}_{2}$ with the strong topology.

The following result was established in [23].

Theorem 12.1. There exists an everywhere dense (in the strong topology) set $\mathscr{B} \subset A$ which is a countable intersection of open (in the weak topology) subsets of $\mathscr{A}$ such that for any $a \in \mathscr{B}$, the following assertions hold:

(1) $\inf \left(f_{a}\right)$ is finite and attained at a unique point $\bar{x} \in X$;

(2) for each $\epsilon>0$, there are a neighborhood $\mathscr{V}$ of a in $\mathcal{A}$ with the weak topology and $\delta>0$ such that for each $b \in \mathscr{V}$, $\inf \left(f_{b}\right)$ is finite, and if $z \in X$ satisfies $f_{b}(z) \leq \inf \left(f_{b}\right)+\delta$, then $\rho(z, \bar{x}) \leq \epsilon$ and $\left|f_{b}(z)-f_{a}(\bar{x})\right| \leq \epsilon$.

Note that an analogous result was established in [9] when $X$ is a Banach space and constraint functions are convex.

Now we present the second main result of [23]. 
414 Solutions of optimal control problems

Let $(X,\|\cdot\|)$ be a Banach space. Consider the set $\mathscr{L}$ of all bounded from below lower semicontinuous functions $f: X \rightarrow \mathbb{R}^{1}$. For the set $\mathscr{L}$, we consider the uniformity determined by the following base:

$$
\mathscr{E}(\epsilon)=\{(f, g) \in \mathscr{L} \times \mathscr{L}:|f(x)-g(x)| \leq \epsilon, x \in X\},
$$

where $\epsilon>0$. Clearly this uniform space is metrizable and complete. We equip the space $\mathscr{L}$ with the topology induced by this uniformity.

For $x \in X$ and $A \subset X$, set

$$
\rho(x, A)=\inf \{\|x-y\|: y \in \mathscr{A}\} .
$$

Denote by $S(X)$ the set of all nonempty closed convex subsets of $X$. For the set $S(X)$, we consider the uniformity determined by the following base:

$$
E_{s}(\epsilon)=\{(A, B) \in S(X) \times S(X): \rho(x, B) \leq \epsilon \forall x \in A \text { and } \rho(y, A) \leq \epsilon \forall y \in B\},
$$

where $\epsilon>0$. It is well known that the space $S(X)$ with this uniformity is metrizable (by a metric $H)$ and complete. We consider the set $S(X)$ endowed with the Hausdorff topology induced by this uniformity. Set $\mathscr{A}=\mathscr{A}_{1} \times \mathscr{A}_{2}$, where $\mathscr{A}_{1}=\mathscr{L}$ and $\mathscr{A}_{2}=S(X)$. For each $a=\left(a_{1}, a_{2}\right) \in \mathscr{A}$ define $\phi_{a}=a_{1}: X \rightarrow \mathbb{R}^{1}, S_{a_{2}}=a_{2} \subset X$ and

$$
f_{a}(x)=a_{1}(x), \quad x \in a_{2}, \quad f_{a}(x)=\infty, \quad x \in X \backslash a_{2} .
$$

Clearly $\inf \left(f_{a}\right)$ is finite for all $a \in \mathscr{A}$.

The following result was established in [23].

Theorem 12.2. There exists an everywhere dense set $\mathscr{B} \subset A$ which is a countable intersection of open everywhere dense subsets of $\mathscr{A}$ such that for any $a \in \mathscr{B}$, the following assertions hold:

(1) $\inf \left(f_{a}\right)$ is finite and attained at a unique point $\bar{x} \in X$;

(2) for each $\epsilon>0$ there are a neighborhood $\mathscr{V}$ of $a$ in $\mathcal{A}$ and $\delta>0$ such that for each $b \in \mathscr{V}, \inf \left(f_{b}\right)$ is finite and if $z \in X$ satisfies $f_{b}(z) \leq \inf \left(f_{b}\right)+\delta$, then $\rho(z, \bar{x}) \leq \epsilon$ and $\left|f_{b}(z)-f_{a}(\bar{x})\right| \leq \epsilon$.

Let $(X,\|\cdot\|)$ be a Banach space,

$$
\rho(x, y)=\|x-y\|, \quad x, y \in X
$$

and let $n \geq 1$ be an integer. We consider the minimization problem

$$
\begin{aligned}
f(x) & \longrightarrow \min , \\
x \in A, \quad g_{i}(x) & \leq 0, \quad i=1, \ldots, n,
\end{aligned}
$$

where $f \in C_{b l}(X), g_{i} \in C_{l}(X), i=1, \ldots, n, A \in S(X)$. 
Set $\mathscr{A}=\mathscr{A}_{1} \times \mathscr{A}_{2}$ where $\mathscr{A}_{1}$ is either $C_{b l}(X)$ or $C_{b}(X)$,

$$
\mathscr{A}_{2}=\mathscr{A}_{21} \times \cdots \times \mathscr{A}_{2 n} \times S(X)
$$

$\mathscr{A}_{2 i}$ is either $C_{l}(X)$ or $C(X)$ or $C_{b l}(X)$ or $C_{l}(X), i=1, \ldots, n$.

For $a_{1} \in \mathscr{A}_{1}$, we set $\phi_{a_{1}}=a_{1}$ and for $a_{2}=\left(g_{1}, \ldots, g_{n}, A\right)$, we set

$$
S_{a_{2}}=\left\{x \in A: g_{i}(x) \leq 0, i=1, \ldots, n\right\} .
$$

and define $f_{a}: X \rightarrow \mathbb{R}^{1} \cup\{\infty\}$ as follows:

$$
f_{a}(x)=\phi_{a_{1}}(x)=a_{1}(x), \quad x \in S_{a_{2}}, \quad f_{a}(x)=\infty, \quad x \in X \backslash S_{a_{2}} .
$$

It is easy to see that for each $a=\left(a_{1}, a_{2}\right) \in \mathscr{A}$ the function $f_{a}: X \rightarrow \mathbb{R}^{1} \cup\{\infty\}$ is lower semicontinuous.

Denote by $\mathscr{A}$ the closure of the set $\left\{a \in \mathscr{A}: \inf \left(f_{a}\right)<\infty\right\}$ in the strong topology. We assume that $\mathscr{A} \neq \varnothing$. In this section, we establish, the following result.

Theorem 12.3. There exists an everywhere dense (in the strong topology) subset $\mathscr{B} \subset A$ which is a countable intersection of open (in the weak topology) subsets of $\mathscr{A}$ such that for each $f \in \mathscr{B}$, the following assertions hold:

(1) $\inf \left(f_{a}\right)$ is finite and attained at a unique point $x_{a} \in X$,

(2) for each $\epsilon>0$, there exist $\delta>0$ and a neighborhood $\mathscr{V}$ of a in $A$ with the weak topology such that for each $b \in \mathscr{V}, \inf \left(f_{b}\right)$ is finite and if $z \in X$ satisfies $f_{b}(z) \leq \inf \left(f_{b}\right)+\delta$, then $\left\|z-x_{a}\right\| \leq \epsilon$ and $\left|f_{b}(z)-f_{a}\left(x_{a}\right)\right| \leq \epsilon$.

Proof. Clearly (A1) and (H2) hold. By Theorem 5.1 and Proposition 5.3, it is sufficient to show that (A2), (A3), and (A4) are valid. We will show that (A2) holds.

Let $f \in \mathscr{A}_{1} \subset C_{b l}(X)$ and $D, \epsilon>0$. There is a positive number $c_{0}$ such that

$$
f(x) \geq-c_{0} \quad \forall x \in X .
$$

Choose a positive number $\epsilon_{0}$ such that

$$
\epsilon_{0}\left(D+4 c_{0}+4\right)<\min \{1, \epsilon\}
$$

and a positive number $\epsilon_{1}<1$ such that

$$
4\left(\epsilon_{1}+\epsilon_{1}\left(1-\epsilon_{1}\right)^{-1}\right)<\epsilon_{0} .
$$

Set

$$
u=\left\{g \in C_{l}(X):(f, g) \in E_{C w}\left(\epsilon_{1}\right)\right\}
$$

(see (12.3)).

Assume that

$$
g \in \mathcal{U}, x \in X, \quad \min \{f(x), g(x)\} \leq D
$$


By (12.19), and (12.3),

$$
|f(z)-g(z)|<\epsilon_{1}+\epsilon_{1} \max \{|f(z)|,|g(z)|\}, \quad z \in X .
$$

It follows from this relation, (12.18), (12.16) and Lemma 4.1 that for all $z \in X$,

$$
\begin{gathered}
|f(z)-g(z)|<\epsilon_{0}+\epsilon_{0} \min \{|f(z)|,|g(z)|\} \\
g(z) \geq f(z)-\epsilon_{0}-\epsilon_{0}|f(z)| \geq-1-2 c_{0}
\end{gathered}
$$

By (12.22), (12.20), (12.17), and (12.16),

$$
\begin{aligned}
|f(x)-g(x)| & <\epsilon_{0}+\epsilon_{0}\left[\min \{f(x), g(x)\}+4 c_{0}+4\right] \\
& <\epsilon_{0}+\epsilon_{0}\left(D+4 c_{0}+4\right)<\epsilon
\end{aligned}
$$

Thus (A2) is valid.

We will show that assumption (A3) holds. Let $\gamma \in(0,1)$. Choose positive numbers $\epsilon(\gamma), \delta(\gamma)$, and $\epsilon_{0}(\gamma)$ such that

$$
\begin{gathered}
\epsilon(\gamma)<\gamma, \quad \epsilon_{0}(\gamma)<\epsilon(\gamma) . \\
d_{C s}\left(g_{1}, g_{2}\right) \leq \epsilon(\gamma) \quad \forall\left(g_{1}, g_{2}\right) \in E_{C s}\left(\epsilon_{0}(\gamma)\right), \quad \delta(\gamma)<8^{-1} \epsilon_{0}(\gamma)^{2} .
\end{gathered}
$$

Assume that $f \in \mathscr{A}_{1} \subset C_{b l}(X), Y \subset X$ is nonempty, $\bar{x} \in Y$, and

$$
f(\bar{x}) \leq \inf \{f(z): z \in Y\}+\delta(\gamma)<\infty .
$$

Define $\bar{f}: X \rightarrow \mathbb{R}^{1} \cup\{\infty\}$ by

$$
\bar{f}(x)=f(x)+\epsilon_{0}(\gamma) \min \{1,\|x-\bar{x}\|\}, \quad x \in X .
$$

Clearly $f \in C_{b l}(X),(f, \bar{f}) \in E_{C s}\left(\epsilon_{0}(\gamma)\right)$, (see (12.2)) and if $f \in C_{b}(X)$, then $\bar{f} \in C_{b}(X)$. It follows from the definition of $\epsilon_{0}(\gamma)$ that $d_{C s}(f, \bar{f}) \leq \epsilon(\gamma)$. Clearly $\bar{f}(x) \geq f(z), z \in X$, and $\bar{f}(\bar{x})=f(\bar{x})$.

Assume that $y \in Y$ and

$$
\bar{f}(y) \leq \inf \{\bar{f}(z): z \in Y\}+2 \delta(\gamma) .
$$

It follows from (12.28), (12.29), (12.27), and (12.26) that

$$
\begin{gathered}
f(y)+\epsilon_{0}(\gamma) \min \{1,\|y-\bar{x}\|\}=\bar{f}(y) \leq \bar{f}(\bar{x})+2 \delta(\gamma)=f(\bar{x})+2 \delta(\gamma) \leq f(y)+3 \delta(\gamma), \\
\min \{1,\|y-\bar{x}\|\} \leq 3 \delta(\gamma) \epsilon_{0}(\gamma)^{-1} \leq \epsilon_{0}(\gamma), \quad\|y-\bar{x}\| \leq \epsilon_{0}(\gamma)<\gamma .
\end{gathered}
$$

Thus (A3) holds.

In order to complete the proof of the theorem, it is sufficient to show that (A4) holds.

In the sequel we need the following auxiliary result (see [23, Proposition 7.1]). 
Proposition 12.4. Let $B(0,1)=\{y \in X:\|y\| \leq 1\}$. Assume that $E$ is a closed convex subset of $X$ such that for all $y \in B(0,1), \inf _{x \in E}\|y-x\| \leq 1 / 8$. Then $0 \in E$.

Hypothesis (A4) will follows from the next lemma.

Lemma 12.5. Let $f \in C_{b l}, A \in S(X)$,

$$
\begin{gathered}
g_{i} \in \mathscr{A}_{2 i}, \quad i=1, \ldots, n, \quad a_{2}=\left(g_{1}, \ldots, g_{n}, A\right), \\
\left\{x \in S_{a_{2}}: f(x)<\infty\right\} \neq \varnothing
\end{gathered}
$$

and let $\epsilon, \delta>0$. Then there are

$$
\left(a_{* 2}\right)=\left(g_{* 1}, \ldots, g_{* n}, A_{*}\right),
$$

where

$$
A_{*} \in S(X), \quad g_{* i} \in \mathscr{A}_{2 i}, \quad i=0, \ldots, n, \quad \bar{x} \in S_{a_{*} 2}
$$

and a nonempty open subset $W$ of $\mathscr{A}_{2}$ with the weak topology such that

$$
\begin{gathered}
\left(A_{*}, A\right) \in E_{s}(\epsilon), \quad\left(g_{* i}, g_{i}\right) \in E_{C s}(\epsilon), \quad i=1, \ldots, n, \\
W \cap\left\{\left(h_{1}, \ldots, h_{n}, B\right) \in \mathscr{A}_{2}:(B, A) \in E_{s}(\epsilon),\left(h_{i}, g_{i}\right) \in E_{C s}(\epsilon), i=1, \ldots, n\right\} \neq \varnothing, \\
f(\bar{x}) \leq \inf \left\{f(x): x \in S_{a_{* 2}}\right\}+\delta<\infty
\end{gathered}
$$

and for all $b_{2} \in \mathcal{W}$,

$$
\bar{x} \in S_{b_{2}} \subset S_{a_{* 2}} .
$$

Proof. For each $r \in(0,1]$, define $A_{r} \in S(X)$ by

$$
A_{r}=\{x \in X: \rho(x, A) \leq r\} \text {, }
$$

define $g_{r i} \in \mathscr{A}_{2 i}, i=0, \ldots, n$ by

$$
g_{r i}(x)=g_{i}(x)-r, \quad x \in X, i=1, \ldots, n,
$$

define

$$
a_{r 2}=\left(g_{r 1}, \ldots, g_{r n}, A_{r}\right)
$$

and put

$$
\mu(r)=\inf \left\{f(x): x \in S_{a_{r 2}}\right\} .
$$

Clearly $\mu(r)$ is finite for all $r \in(0,1]$ and the function $\mu$ is monotone decreasing. There is $r_{0} \in\left(0,8^{-1} \epsilon\right)$ such that $\mu$ is continuous at $r_{0}$. Choose $r_{1} \in\left(0, r_{0}\right]$ such that

$$
\left|\mu\left(r_{1}\right)-\mu\left(r_{0}\right)\right|<16^{-1} \delta .
$$


418 Solutions of optimal control problems

There is

$$
\bar{x} \in S_{a_{r_{1} 2}}
$$

such that

$$
f(\bar{x}) \leq \mu\left(r_{1}\right)+16^{-1} \delta .
$$

Relations (12.45) and (12.43) imply that

$$
f(\bar{x}) \leq \mu\left(r_{0}\right)+8^{-1} \delta .
$$

Set

$$
r_{2}=2^{-1}\left(r_{0}+r_{1}\right)
$$

Clearly

$$
\begin{gathered}
\left(A_{r_{i}}, A\right) \in E_{s}(\epsilon), \quad i=0,1,2, \\
\left(g_{r_{i}}, g_{j}\right) \in E_{C s}(\epsilon), \quad i=0,1,2, j=1, \ldots, n .
\end{gathered}
$$

Choose a positive number $\gamma_{0}$ such that

$$
\gamma_{0}<\min \left\{4^{-1} \delta, 16^{-1},(16)^{-1}\left(r_{0}-r_{1}\right)\right\}
$$

and choose a positive number $\gamma<\gamma_{0}$ such that

$$
\gamma+\gamma(1-\gamma)^{-1}<\frac{\gamma_{0}}{8}
$$

Let $\mathcal{W}$ be the interior of the subset

$$
\prod_{j=1}^{n}\left\{\xi \in \mathscr{A}_{2 j}:\left(\xi_{j}, g_{r_{2} j}\right) \in E_{C w}(\gamma)\right\} \times\left\{B \in S(X):\left(B, A_{r_{2}}\right) \in E_{s}(\gamma)\right\}
$$

of $\mathscr{A}_{2}$ with the weak topology. Set

$$
\begin{gathered}
A_{*}=A_{r_{0}}, \quad g_{* j}=g_{r_{0} j}, \quad j=1, \ldots, n, \\
a_{* 2}=\left(g_{* 1}, \ldots, g_{* n}, A_{*}\right) .
\end{gathered}
$$

By (12.53), (12.44), (12.41), (12.14), (12.40), and (12.39), relation (12.34) holds. Relations (12.53) and (12.48) imply (12.35). In view of the definition of $\mathcal{W}$ (see (12.52)) and (12.48), the relation (12.36) is valid.

Relation (12.37) follows from (12.44), (12.45), (12.42), and (12.53). In order to complete the proofs of the lemma and of the theorem, it is sufficient to show that (12.38) is true for all $b_{2} \in \mathcal{W}$.

Assume that

$$
b_{2}=\left(\xi_{1}, \ldots, \xi_{n}, B\right) \in \mathcal{W},
$$


where

$$
\xi_{j} \in \mathscr{A}_{2 j}, \quad j=1, \ldots, n, \quad B \in S(X) .
$$

It follows from (12.56), (12.55), the choice of $\mathcal{W}$ (see (12.52)), (12.9), (12.39), (12.50), (12.51), (12.53), and (12.47) that

$$
B \subset\left\{z \in X: \rho\left(z, A_{r_{2}}\right) \leq \gamma\right\} \subset\left\{z \in X: \rho(z, A) \leq r_{0}\right\}=A_{r_{0}}=A_{*} .
$$

By (12.56), (12.55), (12.3), the choice of $\mathcal{W}$ (see (12.52)), for each $x \in X, j=1, \ldots, n$

$$
\left|\xi_{j}(x)-g_{r_{2} j}(x)\right|<\gamma+\gamma \max \left\{|\xi(x)|,\left|g_{r_{2} j}(x)\right|\right\} .
$$

Relations (12.58) and (12.51) and Lemma 4.1 imply that for each $x \in X, j=1, \ldots, n$,

$$
\left|\xi_{j}(x)-g_{r_{2} j}(x)\right|<8^{-1} \gamma_{0}+8^{-1} \gamma_{0} \min \left\{\left|\xi_{j}(x)\right|,\left|g_{r_{2} j}(x)\right|\right\} .
$$

We show that for each $j \in\{1, \ldots, n\}$

$$
\left\{z \in X: \xi_{j}(z) \leq 0\right\} \subset\left\{z \in X: g_{* j}(z) \leq 0\right\}, \quad j=1, \ldots, n .
$$

Assume that $z \in X, j \in\{1, \ldots, n\}, \xi_{j}(z) \leq 0$. We show that

$$
0 \geq g_{* j}(z)=g_{r_{0} j}(z)=g_{j}(z)-r_{0} .
$$

We assume the converse. Then $g_{j}(z)-r_{0}>0$ and

$$
g_{r_{2} j}(z)=g_{j}(z)-r_{2}=g_{j}(z)-r_{0}+r_{0}-r_{2} \geq r_{0}-r_{2}=\frac{r_{0}-r_{1}}{2} .
$$

Combined with (12.59) and (12.50), (12.62) implies that

$$
\begin{aligned}
\xi_{j}(z) & \geq g_{r_{2} j}(x)-8^{-1} \gamma_{0}-8^{-1} \gamma_{0}\left|g_{r_{2} j}(z)\right| \\
& \geq \frac{r_{0}-r_{1}}{4}-8^{-1} \gamma_{0}>0,
\end{aligned}
$$

a contradiction. The contradiction we have reached proves the inequality (12.60) for each $j \in\{1, \ldots, n\}$.

Relations (12.60) and (12.57) imply that

$$
S_{b_{2}} \subset S_{a_{* 2}} .
$$

We show that $\bar{x} \in S_{b_{2}}$. By (12.44), (12.14), (12.41)

$$
\bar{x} \in A_{r_{1}} .
$$

Relations (12.65), (12.47), and (12.39) imply that

$$
\bar{x}+2^{-1}\left(r_{0}-r_{1}\right) z \in A_{r_{2}} \quad \text { for each } z \in X \text { such that }\|z\| \leq 1 .
$$


In view of (12.66), (12.56), (12.55), and the choice of $\mathcal{W}$ (see (12.52)) for each $z \in X$ satisfying $\|z\| \leq 1$,

$$
\rho\left(z, 2\left(r_{0}-r_{1}\right)^{-1}(B-\bar{x})\right) \leq 2 \gamma\left(r_{0}-r_{1}\right)^{-1} .
$$

In view of (12.67), (12.51), (12.50), and Proposition 12.4, $0 \in B-\bar{x}$ and

$$
\bar{x} \in B .
$$

Let $j \in\{1, \ldots, n\}$. We show that $\xi_{j}(\bar{x}) \leq 0$. It follows from (12.40), (12.44), (12.41), and (12.14) that

$$
g_{j}(\bar{x})-r_{1}=g_{r_{1} j}(\bar{x}) \leq 0 .
$$

Relations (12.40), (12.69), and (12.47) imply that

$$
g_{r_{2} j}(\bar{x})=g_{j}(\bar{x})-r_{2}=g_{r_{1} j}(\bar{x})+r_{1}-r_{2} \leq-\frac{r_{0}-r_{1}}{2} .
$$

By (12.59), (12.70), and (12.50),

$$
\xi(\bar{x})<g_{r_{2} j}(\bar{x})+8^{-1} \gamma_{0}\left|g_{r_{2} j}(\bar{x})\right|+8^{-1} \gamma_{0} \leq-\frac{r_{0}-r_{1}}{4}+8^{-1} \gamma_{0}<0 .
$$

Thus

$$
\xi_{j}(\bar{x})<0, \quad j=1, \ldots, n .
$$

Relations (12.72), (12.68), (12.55), (12.56), and (12.14) imply that $\bar{x} \in S_{b_{2}}$. Combined with (12.64), this implies (12.38) and the lemma itself.

\section{References}

[1] J. M. Ball and N. S. Nadirashvili, Universal singular sets for the one-dimensional variational problems, Calc. Var. Partial Differential Equations 1 (1993), no. 4, 429-438.

[2] A. Cellina and G. Colombo, On a classical problem of the calculus of variations without convexity assumptions, Ann. Inst. H. Poincaré Anal. Non Linéaire 7 (1990), no. 2, 97-106.

[3] A. Cellina and C. Mariconda, The existence question in the calculus of variations: a density result, Proc. Amer. Math. Soc. 120 (1994), no. 4, 1145-1150.

[4] A. Cellina and S. Zagatti, An existence result in a problem of the vectorial case of the calculus of variations, SIAM J. Control Optim. 33 (1995), no. 3, 960-970.

[5] L. Cesari, Optimization-Theory and Applications, Applications of Mathematics, vol. 17, Springer, New York, 1983.

[6] F. S. De Blasi and J. Myjak, Sur la convergence des approximations successives pour les contractions non linéaires dans un espace de Banach, C. R. Acad. Sci. Paris Sér. A-B 283 (1976), no. 4, 185187 (French).

[7] Generic flows generated by continuous vector fields in Banach spaces, Adv. Math. 50 (1983), no. 3, 266-280.

[8] R. Deville, G. Godefroy, and V. Zizler, Smoothness and Renormings in Banach Spaces, Pitman Monographs and Surveys in Pure and Applied Mathematics, vol. 64, Longman Scientific \& Technical, Harlow, 1993. 
[9] A. D. Ioffe, R. E. Lucchetti, and J. P. Revalski, A variational principle for problems with functional constraints, SIAM J. Optim. 12 (2001/2002), no. 2, 461-478.

[10] A. D. Ioffe and A. J. Zaslavski, Variational principles and well-posedness in optimization and calculus of variations, SIAM J. Control Optim. 38 (2000), no. 2, 566-581.

[11] E. J. McShane, Existence theorem for the ordinary problem of the calculus of variations, Ann. Scoula Norm. Pisa 3 (1934), 181-211.

[12] M. D. P. Monteiro Marques and A. Ornelas, Genericity and existence of a minimum for scalar integral functionals, J. Optim. Theory Appl. 86 (1995), no. 2, 421-431.

[13] B. S. Mordukhovich, Existence theorems in nonconvex optimal control, Calculus of Variations and Optimal Control (Haifa, 1998), Chapman \& Hall/CRC Res. Notes Math., vol. 411, Chapman \& Hall/CRC, Florida, 2000, pp. 173-197.

[14] Ch. B. Morrey Jr., Multiple Integrals in the Calculus of Variations, Die Grundlehren der mathematischen Wissenschaften, vol. 130, Springer, New York, 1966.

[15] S. Reich and A. J. Zaslavski, Convergence of generic infinite products of nonexpansive and uniformly continuous operators, Nonlinear Anal. Ser. A: Theory Methods 36 (1999), no. 8, 1049 1065.

[16] R. T. Rockafellar, Existence and duality theorems for convex problems of Bolza, Trans. Amer. Math. Soc. 159 (1971), 1-40.

[17] L. Tonelli, Fondamenti di Calcolo delle Variazioni. I, Nicola Zanicelli, Bolonia, 1921.

[18]_ Fondamenti di Calcolo delle Variazioni. II, Nicola Zanicelli, Bolonia, 1923.

[19] A. J. Zaslavski, Dynamic properties of optimal solutions of variational problems, Nonlinear Anal. 27 (1996), no. 8, 895-931.

[20] Generic well-posedness of optimal control problems without convexity assumptions, SIAM J. Control Optim. 39 (2000), no. 1, 250-280.

[21] The turnpike property for extremals of nonautonomous variational problems with vectorvalued functions, Nonlinear Anal. 42 (2000), no. 8, 1465-1498.

[22] - Existence of solutions of optimal control problems for a generic integrand without convexity assumptions, Nonlinear Anal. Ser. A: Theory Methods 43 (2001), no. 3, 339-361.

[23] - Generic existence of solutions of minimization problems with constraints, Comm. Appl. Nonlinear Anal. 8 (2001), no. 2, 31-42.

Alexander J. Zaslavski: Department of Mathematics, Technion - Israel Institute of Technology, Haifa 32000, Israel

E-mail address: ajzasl@tx.technion.ac.il 


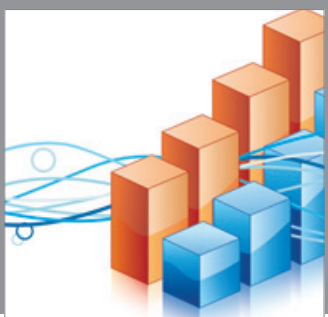

Advances in

Operations Research

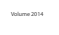

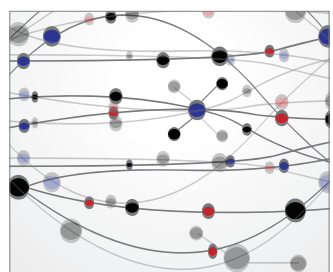

\section{The Scientific} World Journal
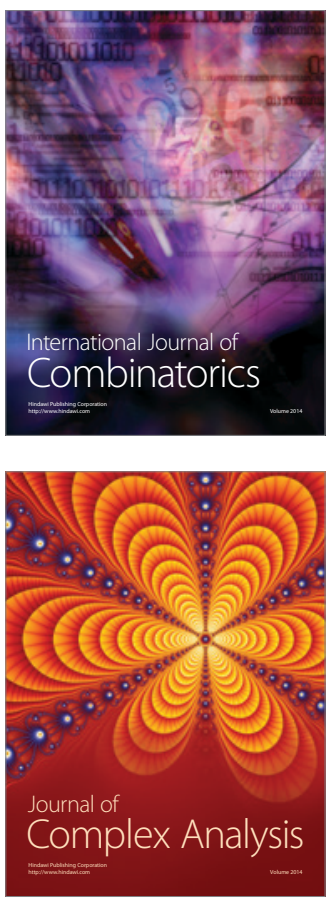

International Journal of

Mathematics and

Mathematical

Sciences
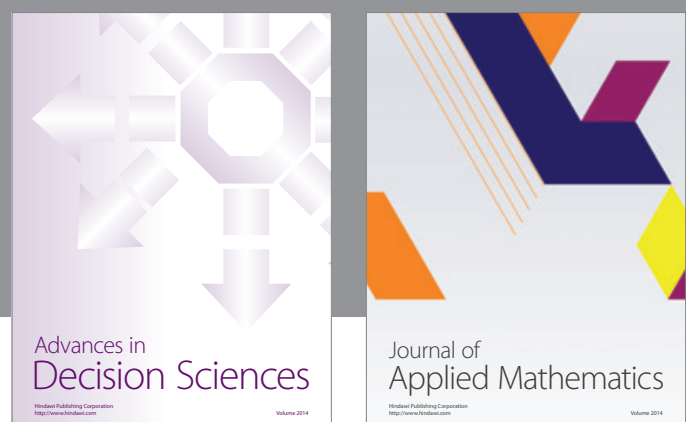

Journal of

Applied Mathematics
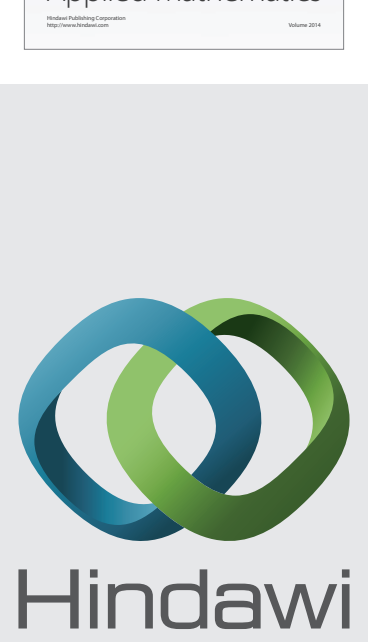

Submit your manuscripts at http://www.hindawi.com
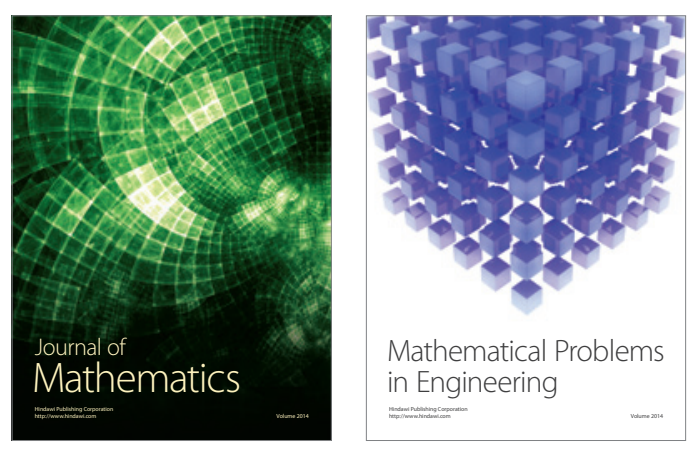

Mathematical Problems in Engineering
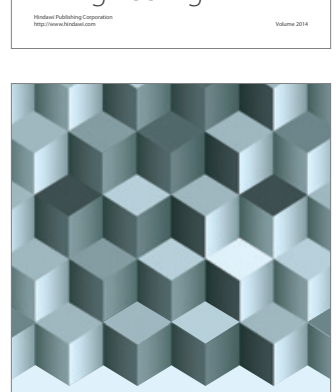

Journal of

Function Spaces
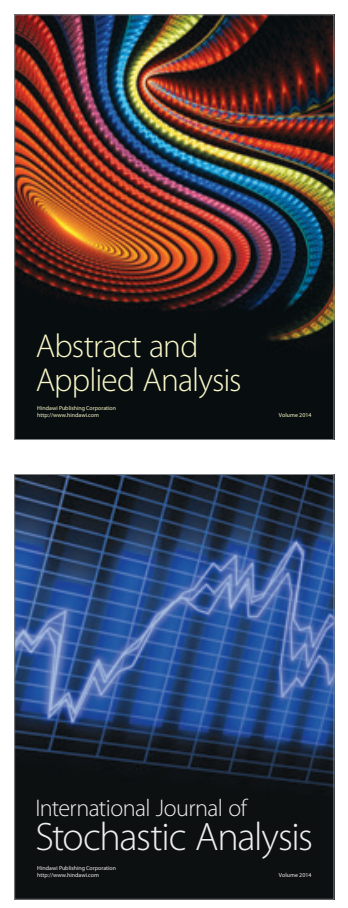

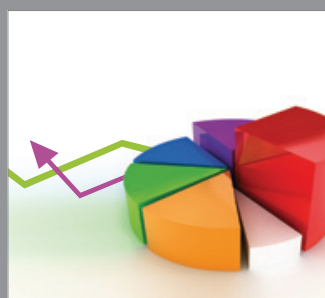

ournal of

Probability and Statistics

Promensencen
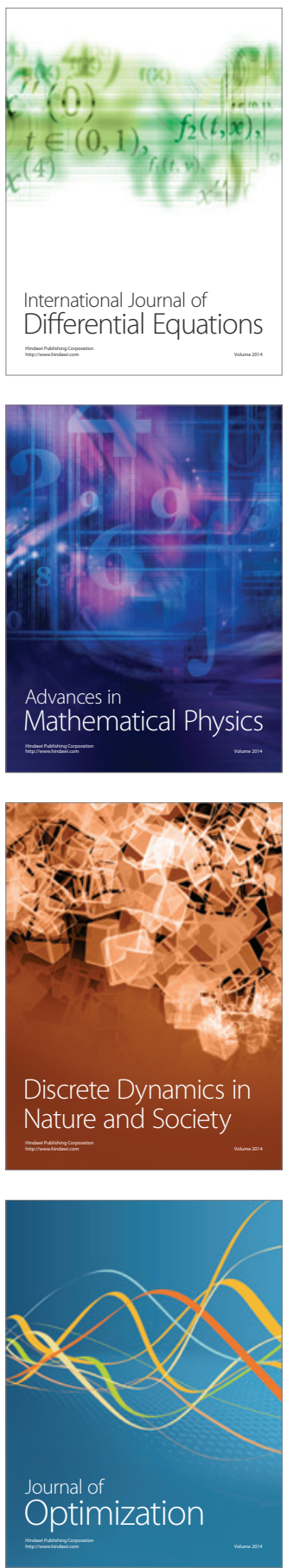